\title{
K-ray Computed Tomography for Medical Imaging
}

HARISH P. HIRIYANNAIAH

$\mathbf{T}$

The mathematical principles of computed tomography

(CT) were first investigated by J. Radon [28] as early

as 1917 and were later extended to complex fields by

Kirillov [22]. The first application of tomography was, surprisingly, in radio astronomy [2], and today, it finds usage in diverse fields such as medical imaging, seismology, and underwater acoustic imaging. In medical imaging, CT has had a tremendous impact in noninvasive diagnostics, surgical planning, etc., as a diagnostic tool. Newer scanning techniques such as the spiral CT are being used that have extended the traditional CT technology. There is vigorous ongoing research in cone-beam CT, in which the mathematical principles are being understood and extended for practical scanner implementations. In this article we will exclusively deal with nondiffractive-transmission CT.

Tomography literally means "slice" or cross-sectional imaging. The central idea is to reconstruct an object from planar integrals of the data. In an $n$-dimensional world, the object is reconstructed from data obtained by integration along hyper-planes intersecting it. In 2-D, the hyper-plane integrals degenerate to line integrals. In this article, we shall limit our discussion to 2-D and 3-D objects. A 3-D object can be examined in two ways.

- The object can be visualized as a stack of 2-D slices.

- The object is examined in its natural 3-D representation.

Various scanning geometries have evolved in 2-D CT. The original CT theory was developed for parallel beam geometries. In the second-generation scanners, fan-beam geometries were used. In this generation of scanners, the detectors were placed on an arc of a circle or a straight line, and the source-detector assembly rotated around the object. In the third-generation scanners, the detectors were placed on a complete circular ring and the $\mathrm{x}$-ray source rotated around the object. The fourth and the current generation of scanners use the spiral or helical CT technology.

Practical 3-D scanners are yet to be built. Various prototypes such as Mayo Clinic's Dynamic Spatial Reconstructor (DSR) and Imatron Inc.'s Electron Beam CT have been developed and show promise, but they are not true 3-D scanners.

In this article we will examine the physical and mathematical concepts of the Radon transform, and the basic parallel beam reconstruction algorithms are discussed. We also develop the algorithms for fan-beam CT, and we briefly discuss the mathematical principles of cone-beam CT.

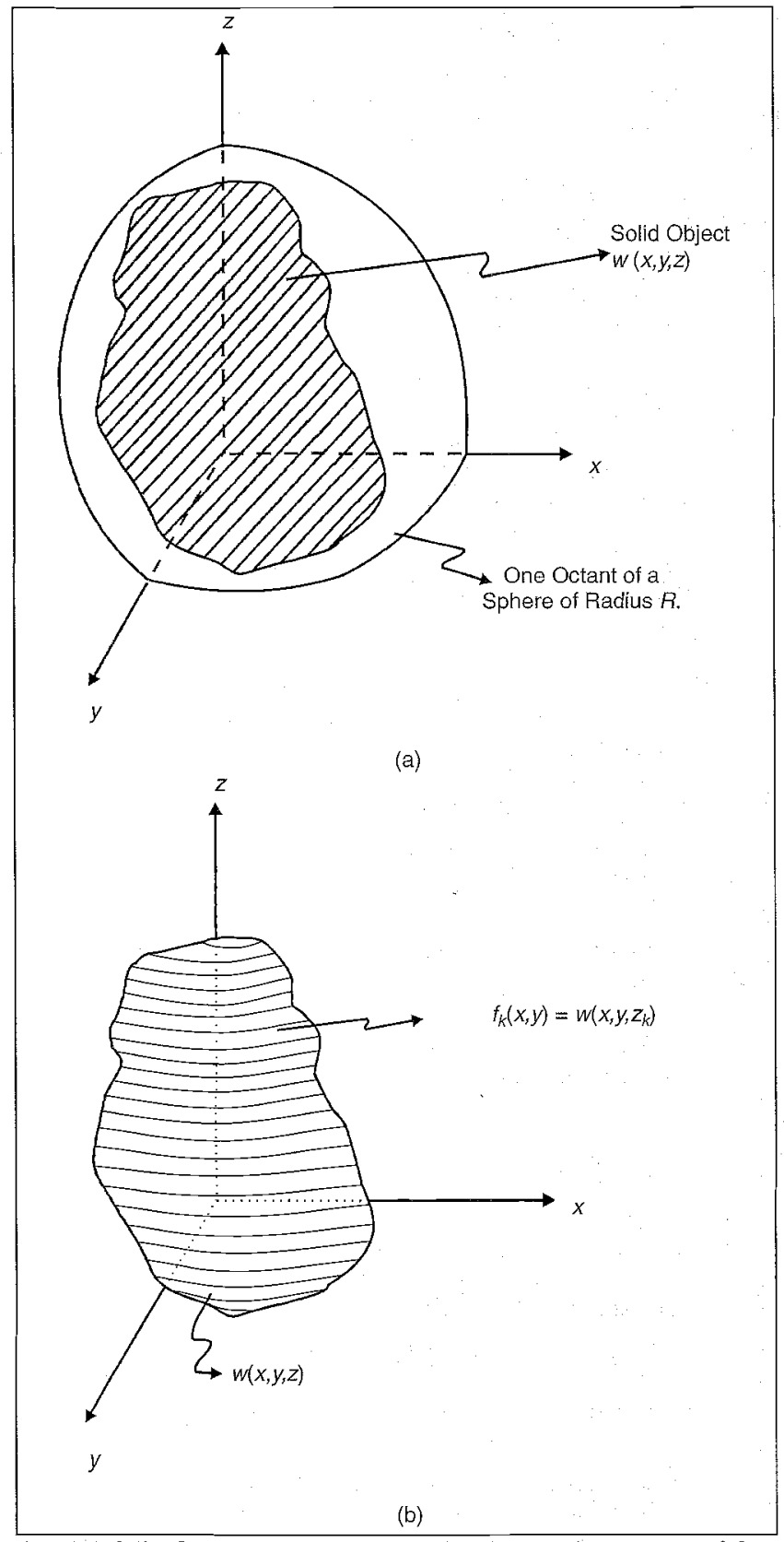

1. (a) A 3-D object within a sphere of radius $R$. One octant of the sphere is depicted. (b) The same object is shown as a stack of slices along the $z$ direction. $f(x, y)=w\left(x, y, z_{k}\right)$ is one such slice at $z$ $=z . k$. 


\section{The 2-D Radon Transform}

Let us consider a real 3-D space, $\mathbb{R}^{3}$. Every point $\mathbf{x} \in \mathbb{R}^{3}$ can be represented by the Cartesian triplet $(x, y, z)$. Let $w(\mathbf{x})$ denote the object with a sphere of support in $R^{3}$ of radius $R$. The object $w$ has a value in the finite range $[a, b]$ and is usually nonnegative, i.e., $a \geq 0$. By fixing the third dimension, $z$, to a particular value, we obtain a 2-D slice $f(x, y)$ of $w(x, y, z)$ (Figure 1(b)). For different values of $z$, we obtain different slices. If these slices are stacked up and properly aligned, then we get a reasonable 3-D stack representation of the object. Ideally, each slice has infinitesimal thickness, but in practical scanners, the thickness is measurable and can be adjusted between a range. If the value of the object in between two slices is needed, then suitable interpolation techniques can be employed by using the neighboring slices [38] (see Figure 1).

Now, let us consider a slice, $f(x, y)=w\left(x, y, z_{k}\right)$, where $z_{k}$ is fixed. We note that this slice is bounded by a circle of radius $R$ (see Figure 2). The $x$ and $y$ coordinates identify the spatial 2-D axes of the slice. Let us also consider a parallel beam of rays intersecting the object. The parallel beam is inclined to the $\mathrm{x}$-axis at an angle $\theta$ and each ray, $M$, can be characterized by its perpendicular distance, $t$, to the origin. A line integration is performed along each ray, $M_{\theta, t}$ and is denoted by:

$$
\dot{P}_{\theta}(t)=\int_{M_{\theta, t}} f(x, y) d s .
$$

where $s$ is along the direction of the ray. $P_{\theta}(t)$ is called the Radon transform of $f(x, y)$. For each fixed $\theta, P_{\theta}(t)$ is a 1-D signal, and hence $\left\{P_{\theta}(\mathrm{t}) \mid \theta \in[0, \pi)\right\}$ gives a complete collection of 1-D projections of the 2-D object $f(x, y)$. We only need $\theta$ to be in the interval $[0, \pi)$, since any interval bigger than this will imply duplication of information. It is easy to see that $P_{\theta}(t)$ is zero outside the interval $[-R, R]$ and the parametric representation of $M_{\theta,}$ is $x \cos \theta+y \sin \theta=t$. Using the Dirac delta function, we have an alternate representation:

$$
P_{\theta}(t)=\iint_{\Re^{2}} f(x, y) \delta(x \cos \theta+y \sin \theta-t) d x d y .
$$

Our objective is to reconstruct the slice $f(x, y)$ from $P_{\theta}(t) . P_{\theta}(t)$ is usually measured by a detector array.

\section{Physics of CT}

We have implicitly assumed that, in X-ray tomography, we have the projection data available to us. How are we measuring this projection data? In X-ray CT used for diagnostic imaging, the energy of the $\mathrm{X}$-rays is approximately $120 \mathrm{keV}$. Any $\mathrm{X}$-ray passing through an object is subject to attenuation either due to photo-electric absorption in the object or due to Compton scatter. If an X-ray beam of a certain energy (and hence wavelength) has a certain intensity measured in terms of the number of photons, $N_{s}$, exiting the source, the number of photons registered at the detector, $N_{d}$, will be lesser, due to absorption and scattering. Let us consider a homogeneous object through which the $\mathrm{X}$-rays are passing. In this case, the combined attenuation factor per unit distance in this material has the relationship:

$$
\frac{N_{s}-N_{d}}{N_{s}}=\mu \Delta l
$$

where $\mu$ is the attenuation factor per unit distance. Solving this differential equation yields us the solution:

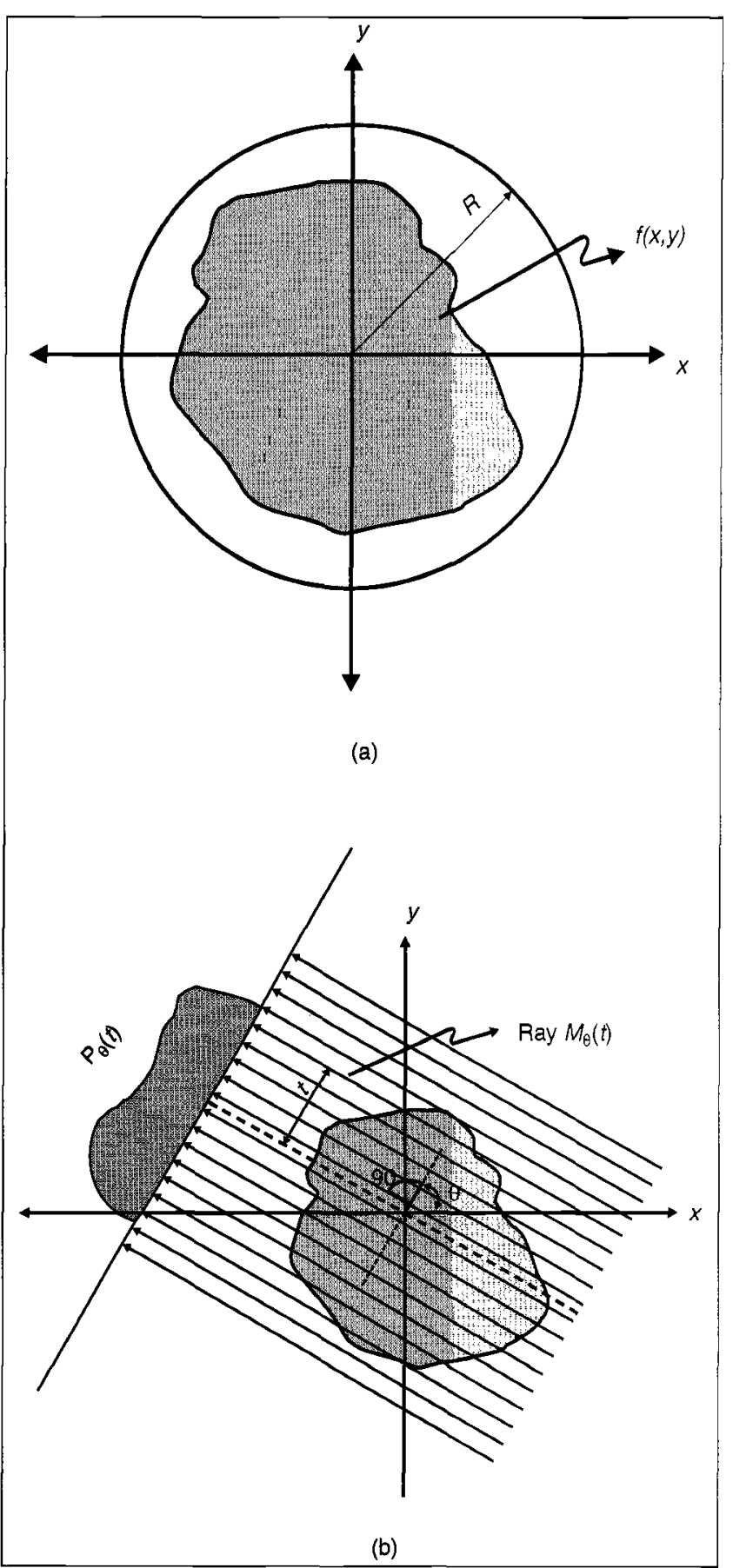

2. (a) A slice, $f(x, y)$, within a circular support of radius $R$. (b) $A$ parallel-beam projection through $f(x, y)$ at an angle $\theta$ from the $x$ axis. $M_{\theta}(t)$ represents a ray passing through $f(x, y)$ at a distance $t$ from the origin at an angle $\theta . P_{\theta}(t)$ is the measured projection data. 


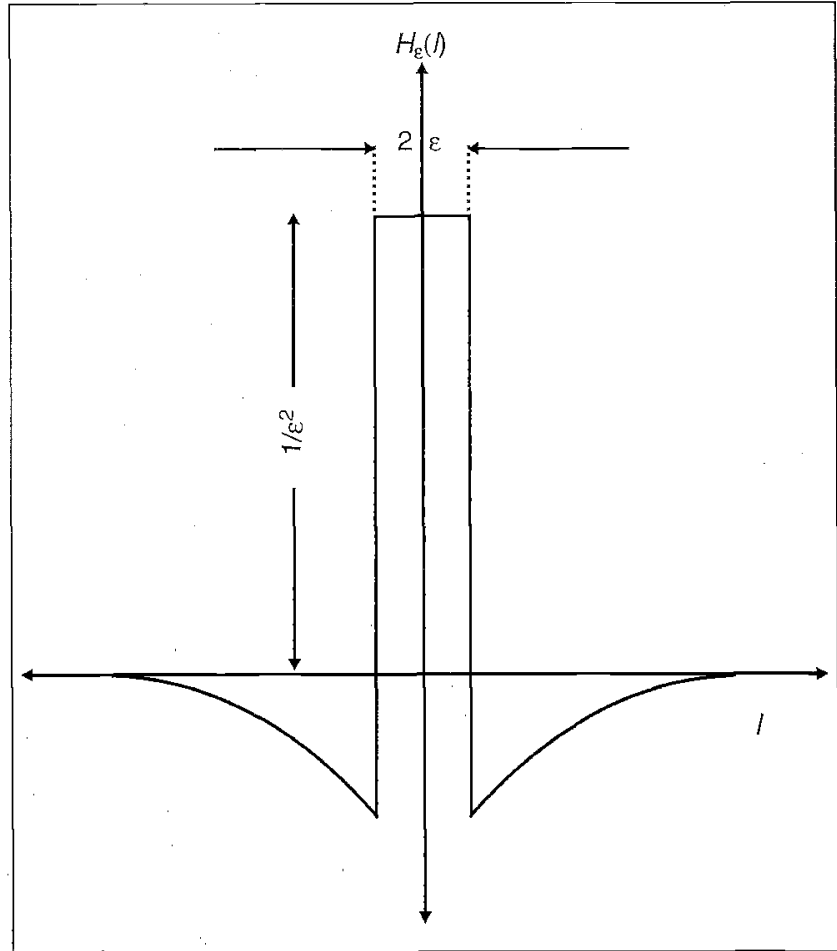

3. $H_{\varepsilon}(l):$ Note the discontinuities at $l=\varepsilon$. The total area under $H_{\varepsilon}(l)$ is zero. The discrete versions of this filter are the Ram-Lak, Horn, and Shepp-Logan kernels.

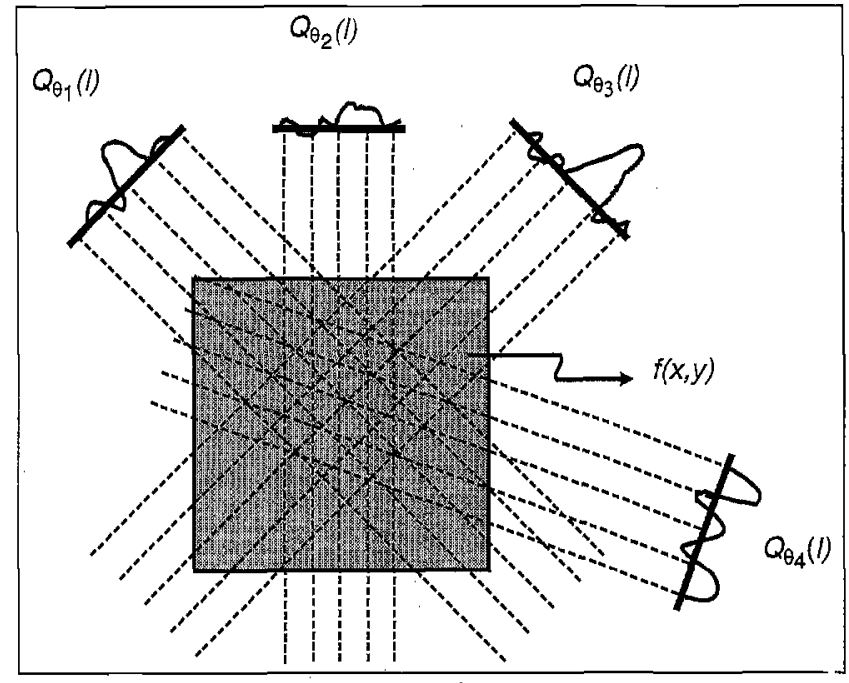

4. $H_{\varepsilon}(l)$ : The projections at $\theta_{1}, \theta_{2}, \theta_{3}, \theta_{4}$, etc., are filtered to obtain $Q_{\theta 1}(l), Q_{\theta 2}(l), Q_{\theta 3}(l), Q_{\theta 4}(l)$, etc. These filtered projections are then smeared (back-projected) onto the image plane.

$$
N(l)=N_{S} e^{-\mu l}
$$

In reality, $\mu$ is not constant since the object is not composed of a homogeneous material and the position dependency is denoted by $\mu(x, y)$. The exponent is now replaced by a line integral and the measured number of photons, $N_{d}$, is given by:

$$
N_{d}=N_{s} e^{-\int \operatorname{pain} \mu(x, y) d s} .
$$

Altematively,

$$
\int \operatorname{pain} \mu(x, y) d s=\ln \frac{N_{3}}{N_{3}}
$$

and the quantity on the left hand side represents the projection data $P_{\theta}(l)$. We are assuming here that the $\mathrm{X}$-rays are monochromatic (or at least the detectors have a very sharp notch filter response to polychromatic X-rays-the detectors are insensitive to scattered polychromatic X-rays). In practical $\mathrm{X}$-ray sources, this is not true. The detector equation is then modified as:

$$
N_{\alpha}=\int N_{s}(E) e^{-\int \operatorname{panh} \mu(x, y, E) d s} d E
$$

where $N_{d}$ is the total number of X-ray photons of all energies registered at the detector, while $N_{s}(E)$ is the number of X-ray photons of energy, $E$, exiting the source, and $\mu(x, y, E)$ is the attenuation factor at location $(x, y)$ for energy $E$. What is the nature of $\mu(x, y, E)$ with respect to $E$ in diagnostic imaging? For most tissues, $\mu$ decreases with increase in $\mathrm{X}$-ray energy. This means that low energy $X$-rays are absorbed more, while high-energy $\mathrm{X}$-rays are passed through, implying that $\mathrm{X}$-ray spectrum of the photons at the detector show a skew in favor of high-energy photons. This is termed as beam hardening. This effect is more noticeable in CT scans of the skull and the artifacts are termed as beam hardening artifacts. In regions near the bones, this effect produces a slight elevation of the measured $\mu[20]$ and results in streaks in the reconstruction.

\section{Inverting the Radon Transform}

In this section we will examine the methods available to us for reconstructing a slice, $f(x, y)$, from its projection data, $P_{\theta}(t)$. The two basic algorithms for parallel beam geometry are filtered back-projection and the Fourier slice method. All the 2-D reconstruction algorithms for various geometries rely on the principles of these two methods.

\section{Filtered Back-Projection}

In two dimensions, it is easy to show $[28,7,31]$ that the inverse Radon transform is reduced to:

$$
f(x, y)=\frac{1}{2 \pi} \int_{0}^{\pi} \int_{-\infty}^{\infty} \frac{1}{(x \cdot \theta-l)} \frac{\partial P_{\theta}(l)}{\partial l} d l d \theta .
$$

The perpendicular distance of the ray, $M_{l, \theta}$, from the point $f(x, y)=l-t=l-\mathbf{x} \cdot \boldsymbol{\Theta}$, where $\boldsymbol{\Theta}=(\cos \theta, \sin \theta)$ and $\mathbf{x}=(x, y)$. There is a singularity at $\mathbf{x} \cdot \boldsymbol{\Theta}=l$ in the inner integral. Using the principal value [31], and assuming that $\partial P_{\theta}(t) / \partial t$ exists and is continuous, it is easy to show that:

$$
f(x, y)=\frac{1}{2 \pi^{2}} \int_{0}^{\pi} \lim _{\epsilon \rightarrow 0} \int_{-\infty}^{\infty} P_{\theta}(l) H_{\epsilon}(\mathrm{x} \cdot \theta-l) d l d \theta
$$


where

$$
H_{\epsilon}(l)= \begin{cases}\frac{1}{\epsilon^{2}} & \text { for }|l|<\epsilon \\ \frac{-1}{l^{2}} & \text { for }|l| \geq \epsilon\end{cases}
$$

The graph of $H_{\epsilon}(l)$ is shown in Figure 3 for a finite nonzero value of $\epsilon$. The inner integral in Equation 9 is a convolution. It can be easily shown that the frequency response of $H_{\epsilon}(l)$ is approximated by $|\omega|$, i.e., $H_{\epsilon}(l)$ acts as a high-pass filter. The outer integral in Equation 9 has the effect of smearing the filtered projection data. If we represent $Q_{\theta}(l)=P_{\theta}(l) * H_{\epsilon}(l)$, then, for every $\theta$, we smear $Q_{\theta}(l)$ on to the image plane $(x, y)$ to obtain $f(x, y)$ (see Figure 4 ). The process of smearing is called back-projection, and hence, this method of reconstruction is called filtered back-projection. We also infer from the above equation that if $P_{\theta}(l)$ is known over the domain $[-R, R] \times$ $[0, \pi)$, we can reconstruct $f(x, y)$ completely. In addition, the following points are evident:

- The contribution of each projection, $P_{\theta}(l)$, to the reconstruction at $(x, y)$ depends on its perpendicular distance, $l$, to the reconstruction point. Except for rays passing very close (within $\varepsilon$ distance) to $(x, y)$, the contribution of the rays varies as the negative inverse of the square of their distances from $(x, y)$

- The weights of the contributions of rays close to $(x, y)$ is such that the sum of all weights is zero, $\int_{\mathscr{R}} H_{\epsilon}(l) d l=0$.

- While $P_{\theta}(l)$ has compact support, i.e., $P_{\theta}(l) \in[-R, R]$, the same is not true for $Q_{\theta}(l)$, since $H_{\epsilon}(l)$ has infinite extent. However, this problem is mitigated by the fact that $H_{\mathrm{e}}(l)$ decays very rapidly $\left(x^{-2}\right.$ decay).

- The $|\omega|$ filter poses some problems as it is not a square integrable function. In practice, $H_{\epsilon}(l)$ is bandlimited and smoothed by appropriate smoothing filters to take care of Gibbsian artifacts [20].

\section{Discrete Implementation of Filtered Back-projection}

In practice, we can gather only a finite number of projections. If the projection angles are evenly spaced in $[0, \pi)$ and we have $N$ such projections. We may approximate the backprojection equation by

$$
f(x, y) \simeq \frac{1}{2 \pi^{2}} \sum_{l=0}^{N-1} Q_{i}(l) \delta \theta
$$

where $Q_{i}(l)$ is the $i$ th filtered projection out of the $[0, N-1]$ and $\delta \theta=\pi / N$.

Also, we have only a finite number of detectors. We will assume, for the moment, that the width of the detectors is negligibly small and spaced apart by a small distance, $T$. We now sample the projection data with sampling distance $T$. Let us represent the sampled version of $P_{\theta}(l)$ as $p_{i}(k)$ and $Q_{\theta}(t)$ as $q_{i}(k)$. To obtain the discretely convolved $q_{i}(k)$ from $p_{i}(k)$, we also have to sample $H \epsilon(t)$. If $H(k)$ is the sampled version, then:

$$
\begin{aligned}
& H(k)=-\frac{w_{k}}{(k T)^{2}} \text { for } k \neq 0 \\
& H(0)=-2 \sum_{k=1}^{\infty} H(k)
\end{aligned}
$$

$H(0)$ is so chosen to satisfy the condition $H_{R} H_{\epsilon}(t) d t=0$ How do we chose $w_{k}$ ? One set of $w_{k}$ can be obtained as follows. We know that the Fourier transform of $H_{\mathrm{\varepsilon}}(t)$ is $|\omega|$, and if we have to sample $H_{\mathrm{\epsilon}}(t)$, then we have to bandlimit $|\omega|$ to avoid aliasing. We choose a filter, $\mathcal{H}(\omega)=|\omega| b_{w}(\omega)$, where:

$$
b_{w}(\omega)= \begin{cases}1 & \omega \mid<W \\ 0 & \text { otherwise }\end{cases}
$$

$b_{n}(\omega)$ is a rectangular window imposed on top of the l $\omega \mid$ filter. It is easy to show that the impulse response of $\mathcal{H}(\omega)$ is given by:

$$
h(n T)= \begin{cases}\frac{1}{4 T^{2}} & n=0 \\ 0, & n \text { even } \\ -\frac{1}{n^{2} \pi^{2} T^{2}} & n \text { odd }\end{cases}
$$

This impulse response is very widely used and is commonly referred as the Ram-Lak kernel, after Ramachandran and Lakshminarayanan [29]. Two other kernels commonly used are the Shepp-Logan kernel:

$$
w k=4 k^{2} /\left(4 k^{2}-1\right)
$$

and the Horn kernel [16]:

$$
w_{k}=1 \text {. }
$$

The Horn kernel is obtained by considering the trapezoidal rule for numerical integration, while the Shepp-Logan kernel is obtained by considering a numerical integration formula, which accounts for the singularity in the inverse Radon transform. From a signal processing perspective, choosing

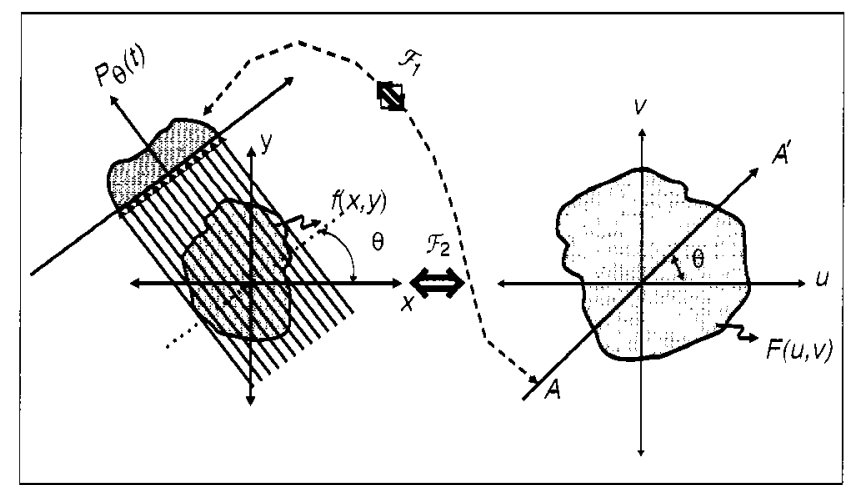

5. This picture depicts the relationship between the 1-D Fourier transform $S_{\theta}(\omega)$ of $P_{\theta}(t)$ and the 2-D Fourier transform of $F(u, v)$ of $f(x, y) . \omega$ and $\theta$ completely describe each point $(u, v)$ in the Fourier plane. i.e., $(u, v)=(\omega \cos \theta, \omega \sin \theta)$. 
the Horn or Shepp-Logan kernel is equivalent to using a smoothing window on the $1 \omega \mid$ filter. In practice, the Ram-Lak kernel is used with Hamming and other smoothing windows.

\section{Some Implementation Issues}

- The projection data is not truly bandlimited and hence there is going to be some amount of aliasing because of sampling. One way to minimize the aliasing is to select a sampling interval so that a major portion of the energy spectrum of the projections is obtained unaliased. Assuming that the actual tail of the spectrum of the projection data tapers towards zero rapidly, it may not be a bad idea to specify that the sampling frequency accommodate all frequencies that contribute to $X \%$ (say 99\%) of the energy. But this is subject to detector size limitations.

- The bandwidth of the windowed |w| filter must be chosen so that it is much more than the bandwidth of the projection data. This is not an easy task, and conflicts easily arise. Choosing a much larger bandwidth for the filter implies that the projection data must be finely sampled, to which there are practical limits because of detector size limitations.

- We have implicitly assumed that projections are sampled using the Kronecker impulse train. However, detectors have a finite rectangular spatial aperture and the resulting effect is the implicit low-pass filtering of the projection data before sampling. These effects should be carefully considered while choosing a smoothing window for the $|\omega|$ filter (see sidebar).

- The Ram-Lak filter [16] is perhaps the best filter in terms of fine spatial frequency resolution, but it is sensitive to noise and Gibbs artifacts because of the discontinuity in the frequency domain. The Horn filter tends to blur edges while

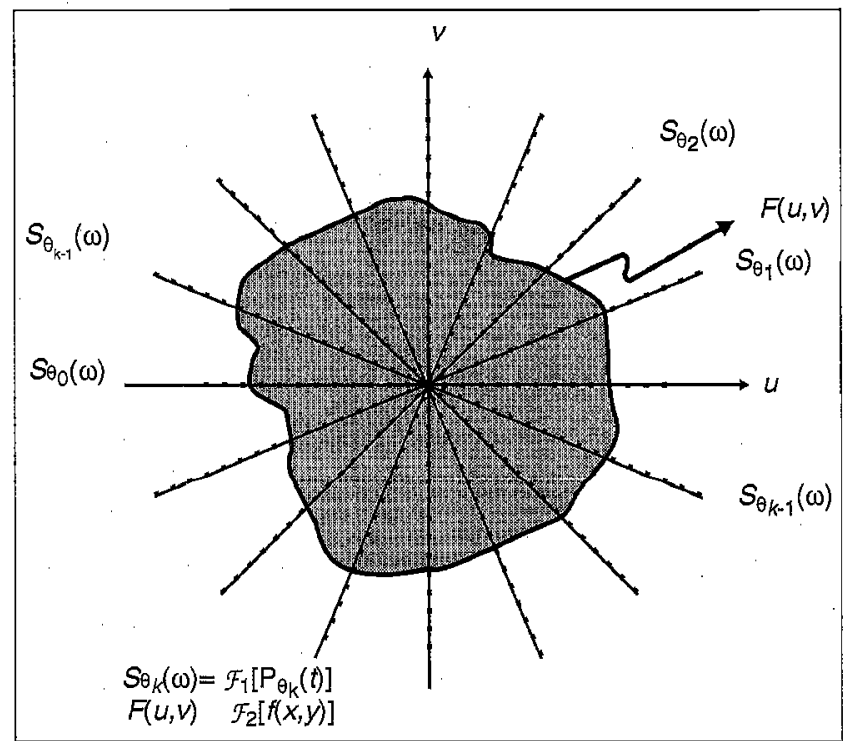

6.Using the Fourier slice theorem, if we try to reconstruct the image by estimating $F(u, v)$ along radial lines $S_{\theta}(\omega)$, we observe non-uniform sampling, with dense clusters of samples near the origin and sparse samples away from the origin. This leads to gross interpolation errors in the higher frequencies. suppressing noise. The Shepp-Logan filter lies in between in performance. In practice, the use of various smoothing windows tends to blur edges, and to obtain a compromise, one uses various linear combinations of these smoothing windows to suit different applications. This is evident in the various filters that an operator can choose during reconstruction in practical scanners.

- We have assumed that the projection data have a continuous derivative $\partial P_{\theta}(t) / \partial t$. If there are regions that violate these assumptions (which are absolutely required for inverse Radon transforms), then these regions will contribute to overshoot and other Gibbs-like artifacts [33].

- During back-projection (see Figure 7), for a given angle $\theta_{i}$, the contribution to the pixel $(i, j)$ lies between the filtered samples $q_{i}(k)$ and $q_{i}(k+1)$. Some sort of interpolation has to be performed. Various interpolation techniques, such as zero-order, linear, spline, and Lagrange methods may be used. The choice will depend upon a priori knowledge of the nature of $f(x, y)$, the amount of computation that can be practically used for the given application, and the visual quality that can be achieved for a particular interpolation method. Linear interpolation is very popular, while cubic spline and other sophisticated curve fitting methods are very good at isolating sub-pixel edges. Other signal processing techniques such as subsampling, super-sampling and pre-interpolation (by zero-padding in the frequency domain) $[20,26,6]$ methods may also be employed, and these methods may have better computational efficiency than traditional interpolation methods.

- Care should be exercised during the filtering of projection data using FFT. Noting that the convolution in DFT domain is circular, while an aperiodic convolution is actually needed, the projection data should be sufficiently zeropadded to remove interperiod artifacts [26].

\section{Direct Fourier Transform Method}

One of the important properties of the Radon transform, $P_{\theta}(t)$, of an object, $f(x, y)$, is its relationship to the Fourier transform, $\mathcal{F}(u, v)$ of $f(x, y)$, usually termed as the "Fourier Slice Theorem." Recalling that:

$$
\mathcal{F}(u, v)=\iint_{R^{2}} f(x, y) e^{-j(u x-v y)} d x d y
$$

and defining the Fourier transform $S_{\theta}(\omega)$ of $P_{\theta}(l)$ as

$$
S_{\theta}(\omega)=\int_{\Omega} P_{\theta}(l) e^{-j \omega l} d l
$$

let us examine $\mathcal{F}(u, 0)$ :

$$
\mathcal{F}(u, 0)=\int_{R}\left[\int_{R} f(x, y) d y\right] e^{-j u x} d x
$$

The inner integral is $P_{\theta}=0(x)$ and the outer integral calculates its Fourier transform $S_{\theta=0}(\omega)$, i.e., $\mathcal{F}(u, 0)=S_{\theta=0}(\omega)$. By considering a system of coordinates $(t, s)[20]$ rotated by an 


\section{Rebinning}

In fan-beam reconstruction, $f(x, y)$ was reconstructed directly from the back-projection of the filtered fanbeam data. Another way to accomplish the reconsiruction is to re-sort the fan-beam data into equivalent parallel-beam data, and use the standard parallel beam filtered back-projection algorithm. From our previous discussion, we have, for an equiangular fan-beam geometry, $\theta=\beta+\gamma$ and $t=D \sin \gamma$. Then, for every fanbeam ray $R_{\beta}(\gamma)$, we have the corresponding parallelbeam measurement $P\{\beta+\gamma\}(D \sin \gamma)$. If $\beta$ and $\gamma$ are sampled uniformly, i.c., $\beta=m \Delta \beta$ and $\gamma=n \Delta \gamma$, we have the sampled version $R_{m, \Delta \beta}(n \Delta \gamma)=P_{m \Delta, \beta+m}(D \sin \Delta \gamma)$. We note that $P$ is nonuniformly sampled in both dimensions, $l$ and $\theta$. We have to resort to interpolation to get a uniformly sampled $(l, \theta)$ domain. We can alleviate the interpolation effort by constraining $\Delta \beta=\Delta \gamma=\alpha$. Given the geometries of the detector array and the $X$-ray source, this is not an unreasonable constraint. We then have $R_{r n \alpha}(n \alpha)=P_{(m \cdot n)} \alpha(D \sin n \alpha)$. We now have a faster re-sorting (rebinning) algorithm, where $n$th ray in the $m$ th fan beam maps to $a$ ray in the $(m+n)$ th parallel projection.

In the above strategy, it is quite possible that some projections will have more samples and some will have less. However, by using suitable interpolation, it is possible to recover the required number of uniform samples in the parallel projection domain. This rebinning method is sensitive to interpolation crrors. We note that, in the case of cone-beam CT, the calculation $G(\beta, \Phi)=F(\beta, \Phi \cdot \beta)$ implies a similar rebinning strategy accomplished through interpolation.

angle $\theta$ from the $(x, y)$ coordinates, we can generalize the above relation to show that:

$$
S_{\theta}(\omega)=\mathscr{F}(\omega \cos \theta, \omega \sin \theta) .
$$

From Figure 5 and the equation above, we infer that if we calculate the Fourier transform of $P_{\theta}(l)$, for all $\theta$, then it yields the Fourier transform $\mathcal{F}(u, v)$ of $f(x, y)$ (see Figure 6). We can then easily obtain $f(x, y)$ by taking the inverse Fourier transform of $\mathcal{F}(u, v)$.

\section{Issues in the Direct Fourier Transform Method}

The Fourier slice theorem, at first glance, looks attractive for tomographic reconstruction. However, we must note the following points about Fourier slice reconstruction.

- The Fourier transform $S_{\theta}(\omega)$ of $P_{\theta}(t)$ gives us the values of the Fourier transform $\mathcal{F}(u, v)$ of $f(x, y)$ along a radial path at angle $\theta$ in the $(u, v)$ domain (see Figures 6 and 8). For equiangular $\theta$, we obtain values of $\mathcal{F}(u, v)$ along radial paths in concentric circles. These values have to be then interpo-

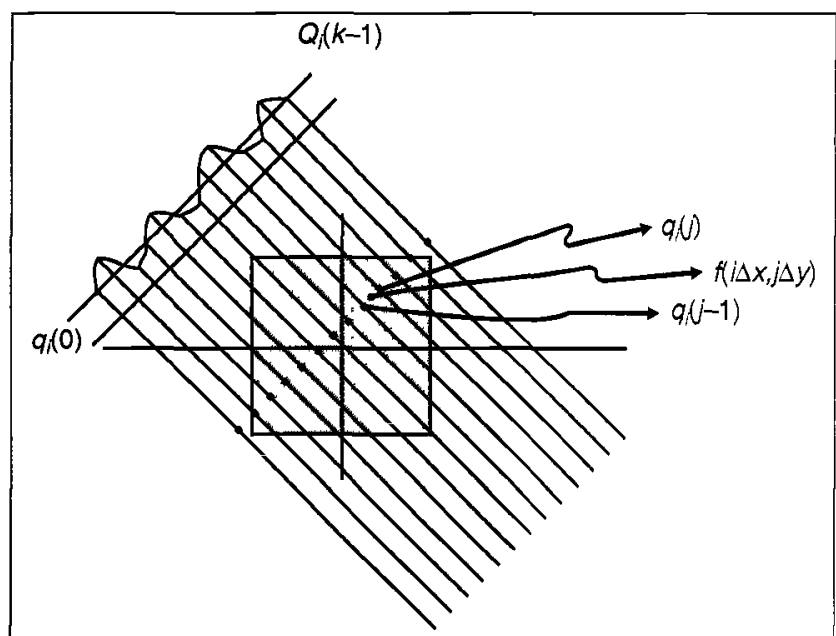

7. Back-projection. The back-projected value at a specified location ( $i \Delta x, j \Delta y$ ) can be obtained by interpolation. The easiest method is to linearly interpolate between two neighboring values.

lated onto a rectangular grid. The interpolation errors will not be constant. Toward the origin, there will be a dense collection of samples and interpolation errors will be small. In the high-frequency regions, the samples are sparse and interpolation errors will be high. Consequently, edges and other high-frequency spatial content in the signal $f(x, y)$ will be distorted.

- The choice of an interpolation rule is not clear since this has to be done in the frequency domain. Surface fitting methods $[17,39]$ are probably the best method, but these methods tend to be computationally very expensive.

- As noted earlier, the Fourier transform of the projections may not be bandlimited. To prevent aliasing effects, we will have to either bandlimit the projection Fourier transforms prior to interpolation in the $\mathcal{F}(u, v)$ domain or bandlimit $\mathcal{F}(u, v)$ after interpolation.

\section{Fan-Beam CT}

In parallel-beam geometry, for each angle, $\theta$, the source (and the detector) must have a translation motion (to cover the $t$ dimension). It is very cumbersome to develop such $\mathrm{X}$-ray source-detector systems. In addition, very large scanning times and undue exposure to ionizing radiation for extended periods are involved. Fan-beam tomography alleviates some of these problems. In this set-up, the X-ray beam is collimated so that a thin, planar fan beam of rays extends from the $\mathrm{X}$-ray source and passes through the object before being collected by a detector array. The X-ray source then rotates around the object. (Or, in an equivalent motion. the object rotates about an axis perpendicular to the fan beam, as is normally done in industrial CT scanners). In each source position, the fan beam completely covers the object (see Figure 9). There are two major detector configurations (Figure 10):

- Equiangular fan beam-the detector array lies on a circular arc (or a complete circle as in present-day scanners) such that the angle subtended between two detectors is constant. 


\section{Sinograms}

What are sinograms? If we consider the Radon space and restrict it to the extent of the object $[-R, R] \times[0, \pi)$, it completely covers a circle in the $(l, \theta)$ domain. If the same domain is represented in Cartesian coordinates, as shown in figure 13 , it covers a rectangular region. This kind of pictorial representation is called a sinogram. Why is it called a sinogram? Consider the Radon transform equation (Equation 2). If $f(x, y)$ corresponds to a delta function $\delta\left(x-x_{u}, y-y_{u}\right)$, then it is easy to see that the corresponding representation in the sinogram will be a sinusoidal function.

Sinograms are very useful in visualizing the projection data $P_{\theta}(l)$ and the corresponding data mapping in fan-beam geometries. Figure 13 also shows the extent of overlap in the case of data collection from limitedangle fan-beam projection (the angle of coverage for $\beta$ being $\left.180^{\circ}+2 \gamma_{m}\right)$. For the case of $\beta \in[0, \pi)$, the sinogram shows that there are regions (marked $A$ ) where data has been measured twice and regions (marked B) where there is no measurement at all. To get complete coverage at least once, we must increase the scanning angle for $\beta$ to at least $180^{\circ}+2 \gamma_{m}$. This, however, increases the overlapped regions (A). To compensate for the overlapped data, a suitably smoothed one-zero windowing filter is applied to the fan-beam data before reconstruction.

- Equidistant collinear detector fan beam-the detectors lie on a straight line and are equidistant from each other.

In order to understand the reconstruction algorithms for these two configurations, let us first examine a ray-sampling configuration in generalized coordinates.

\section{Generalized Sampling Coordinates}

In the previous sections, we based our reconstruction on the fact that our uniform sampling coordinate systen is $(t, \theta)$. However, this may not be a suitable coordinate system for data acquisition. Let us assume that we have uniform sampling in our acquisition coordinate system, $(\xi, \eta)$. We may now write our reconstruction formula in these coordinates as follows [161:

$$
f(r, \phi)=\frac{1}{4 \pi^{2}} \lim _{\epsilon \rightarrow 0} \iint H_{\epsilon}(t) P_{\theta}(l-t) /(l, \theta, \xi, \eta) d \xi c h .{ }^{(22)}
$$

$l$ and $t$ are dependent on $r$ and $\phi$. Appropriate limits of integration are used for $(\xi, \eta)$ space. $J$ is the Jacobian of the transformation from $(\xi, \eta)$ space to $(l, \theta)$ space and is given by:

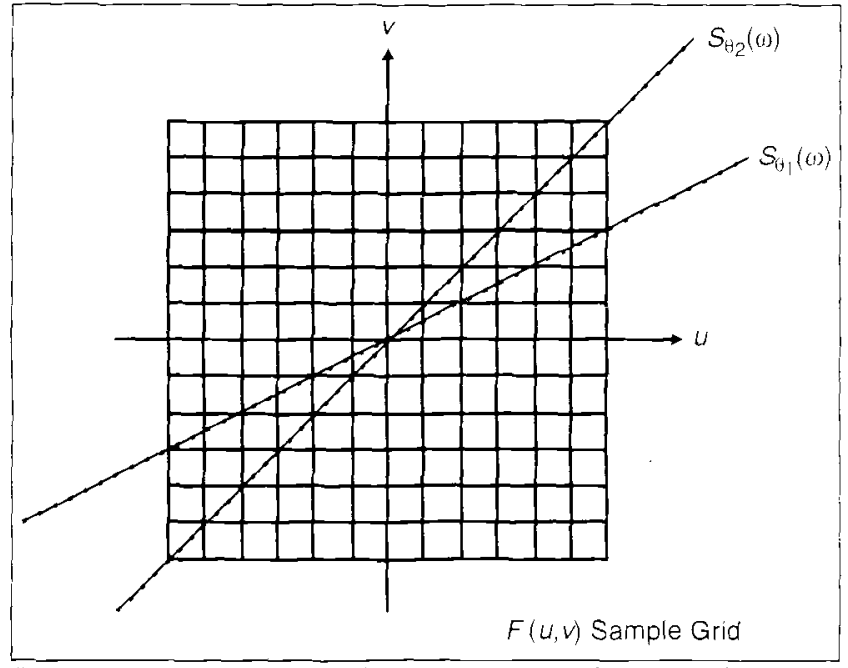

8. Direct Fourier method. This picture shows the interpolation problems in the $(u, v)$ domain due to nonuniform sampling. The interpolation errors increase with increasing of and $b$ :

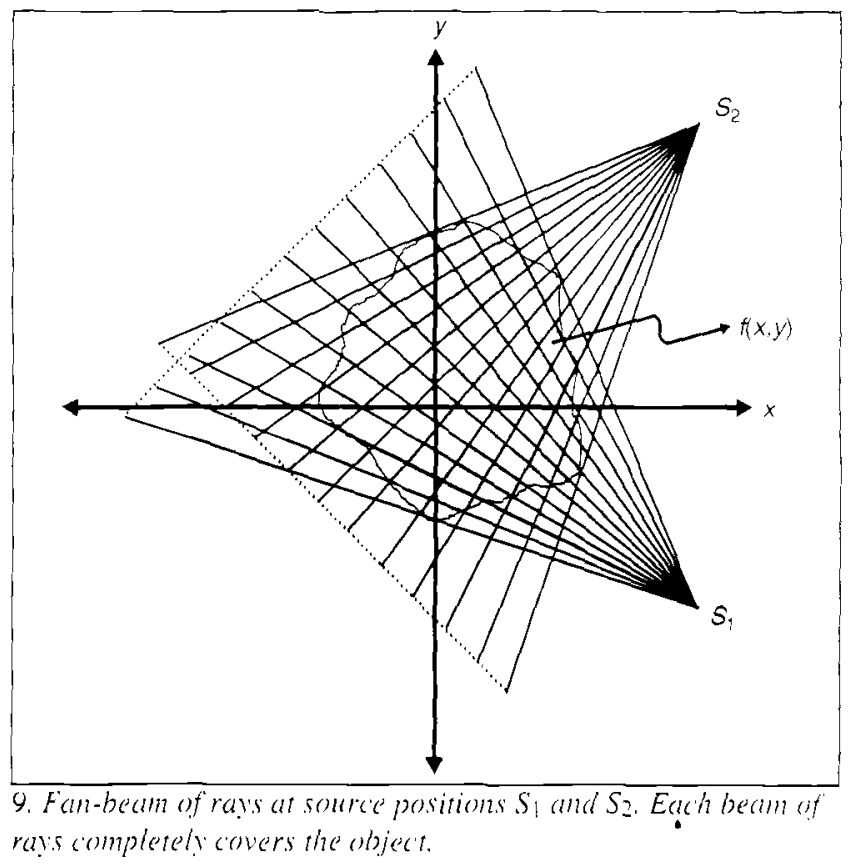

$$
J=\frac{\partial t}{\partial \xi} \frac{\partial \theta}{\partial \eta}-\frac{\partial \theta}{\partial \xi} \frac{\partial l}{\partial \eta}
$$

Uniform sampling in $(\xi, \eta)$ space usually does not translate to uniform sampling in $(l, \theta)$ space, and intuitively, we see that the sampling density in the latter space is inversely proportional to $J$. In the equation above, we may represent the inner integral as:

$$
g(r, \phi, \eta)=\lim _{\xi \rightarrow 0} \int H_{\varepsilon}(1-t) P(\xi, \eta) J d \xi
$$

and the equivalent back-projection equation can be written as: 


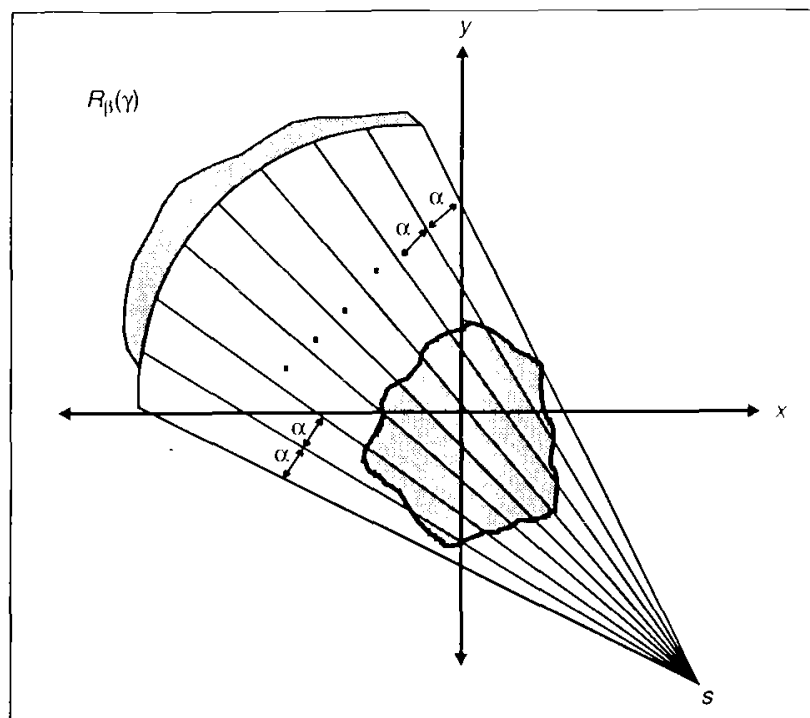

(a)

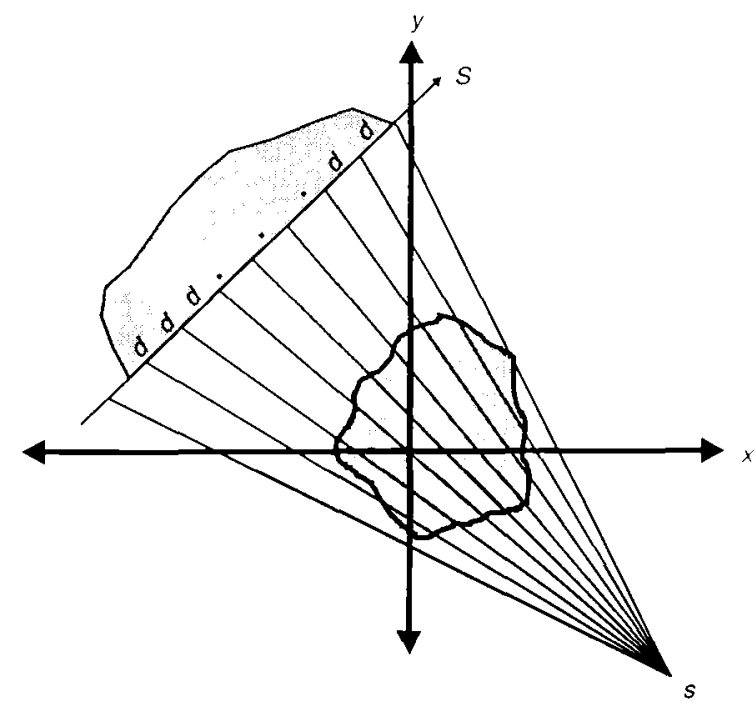

(b)

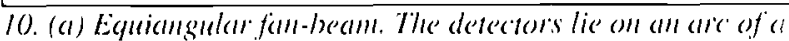
circle with angular spacing $\alpha$. (b) Equidistant detector fan-becam. The detectors are collinear with uniform sampling distances d.

$$
f(r, \phi)=\frac{1}{4 \pi^{2}} \int g(r, \phi, \eta) d \eta .
$$

In general, $(l-t)$ is a function of $r, \phi, \xi$ and $\eta$. However. for fan-beam geometries, $(l-t)$ will lead to separable functions and the evaluation of $g$ becomes more tractable.

\section{Reconstruction from Equiangular Fan-Beam Projections}

In this configuration, the detectors are placed either on an are of a circle or on a complete circular ring and anc equaly spaced so that the angle subtended between two detectors at the source is constant (saly $\alpha$ ) (see fingure 11 ). If the deteetom are on an anc of a circte, then the sourec and the detector ats-

\section{Detector/Collimator Aperture Effects}

In the filtered back-projection algorithm, we have assumed that the detector width is negligible and sampling is done essentially by a Kronecker delta train. In reality, the combined geometry of the collimators and the detectors results in a finite aperture. Let us denote the aperture function as:

$$
a(l)= \begin{cases}1 & |l| \leq \frac{T_{d}}{2} \\ 0 & \text { otherwise }\end{cases}
$$

where $T_{d}$ is the width of the aperture. The Fourier transform of this function is given by $A(\omega)=T_{d} \operatorname{sinc}\left(\omega T_{d} / 2\right)$. The observed projection is essentially a convolution of $a(l)$ with $P_{\theta}(l) . A(\omega)$ is a low-pass filter. By imposing a rectangular window in the frequency domain, we may approximate the aperture function by:

$$
A^{\prime}(\omega)=\left\{\begin{array}{cc}
T_{d} \operatorname{sinc}\left(\omega T_{d} / 2\right) & |\omega| \leq \omega_{t p} \\
0 & \text { otherwise }
\end{array}\right.
$$

where $\omega_{l p}$ is the low-pass filter bandwidth corresponding to the first zero in the sinc function. Now, let the sampling interval be $T_{s}$. The Kronecker impulse train can be represented as and its Fourier transform is given by $K(\omega)=\frac{2 \pi}{T_{z}} \sum_{n=-\infty}^{\infty} \delta\left(\omega-\frac{2 \pi n}{T_{s}}\right)$. Hence, the sampled projection data $p_{\theta i}(j)=\mathcal{F}^{\prime}\left[\left(S_{\theta} ;(\omega) A^{\prime}(\omega)\right) * K(\omega)\right]$. It is quite evident from the above that, to avoid aliasing effects, $T_{s} \leq \frac{T_{d}}{2}$. This implies that we have sample at least twice within the one detector width-a rather nonintuitive finding! In parallel-beam CT configuration and fourth-generation (fixed-detector fan beam) CT, this is effectively accomplished by sampling the detectors twice per source position.

For third-generation (rotating-detector fan-beam) scanners, a technique called quarter-detector offset is used. Recall that, for fan-beam reconstriction, we really need the data through $180^{\circ}+2 \gamma_{\text {in }}$ angle only and collecting it through $360^{\circ}$ results in redundant measurement. The detector array is usually symmetric with respect to the line joining the $X$-ray source and the origin. However if the detector array is offset (translated to the left or right of the origin) by $1 / 4$ th of the detector spacing, then the rays in opposite views are unique. If data is collected over $360^{\circ}$, it effectively doubles the sampling frequency. semblies move together in a circular trajectory atround the object. This setup represents the second generation of ("T" scanners. The third generation of ("T scanners hats a cilcular ring

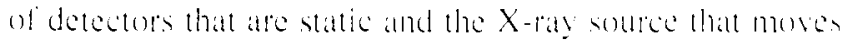
around the ohject atong a circulat thajectory. The advallages with this setup are: 


\section{Noise in CT Reconstructions}

The detector measurements, depending upon the type of detector used, are directly or indirectly dependent on the photon counting statistics that are inherently Poisson in nature. For very large counts, we may safely assume that the statistics are stationary and white. This is not strictly true since the presence of a structure such as a bone in the object significantly alters the counting statistics in regions of the projection data where the rays travel through it, in comparison with the projection of areas in the object where the rays pass only through soft tissue. In addition to these effects we also encounter shot noise in the detectors and quantization noise while sampling the detector output. For the sake of simplicity and a tractable noise model, we will pursue with the white noise assumption. In the continuous domain, the noisy projection data $P_{\theta}{ }^{\eta}(l)$ is modeled by:

$$
P_{\theta}^{\eta}(l)=P_{\theta}(l)+\eta_{\theta}(l)
$$

where $\eta_{\theta}(l)$ models the additive white Gaussian noise [15, 30]. The autocorrelation of this process is given by:

$$
\mathbb{R}_{\mathrm{n}}\left(l_{1}, l_{2}, \theta_{1}, \theta_{2}\right)=S_{o} \delta\left(l_{1}-l_{2}\right) \delta\left(\theta_{1}-\theta_{2}\right) .
$$

The reconstructed object $f^{\prime}(x, y)$ is obtained as:

$$
f^{\prime}(x, y)=f(x, y)+f^{\eta}(x, y)
$$

where $f^{\prime}(x, y)$ is the noisy reconstruction and $f^{\prime}(x, y)$ is the noise component in the reconstruction and is given by $f^{\prime}(x, y)=1 / 2 \pi \int_{0}^{\pi} \eta_{\theta}(l) * H_{\mathrm{\varepsilon}}(l) d \theta$, where * denotes convolution. We would like to characterize the nature of the autocorrelation of the noisy image as follows:

$$
\begin{aligned}
R\left(x_{1}, y_{1}, x_{2}, y_{2}\right)= & E\left\{f^{\eta}\left(x_{1}, y_{2}\right) f^{\eta}\left(x_{2}, y_{2}\right)\right\} \\
= & \frac{S_{0}}{4 \pi^{2}} \int_{0}^{\pi} \\
& \int_{0}^{\pi} E\left\{\left[\eta_{\theta_{1}}\left(l_{1}\right)^{*} H_{\epsilon}\left(l_{1}\right)\right]\left[\eta_{\theta_{2}}\left(l_{2}\right)^{*} H_{\epsilon}\left(l_{2}\right)\right]\right\} \\
& d \theta_{1} d \theta_{2} \\
& =\frac{S_{0}}{4 \pi^{2}} \int_{0}^{\pi} H\left(l_{2}-l_{1}\right) d \theta
\end{aligned}
$$

Here, $H(l)=H_{\epsilon}(l) * H_{\mathrm{\epsilon}}(l)=\mathcal{F}^{1}\left\{|\mathcal{H}(\omega)|^{2}\right\} ; \mathscr{H}(\omega)=$ $\mathcal{F}\left\{H_{\epsilon}(l)\right\}$; and $\mathcal{F}$ is the Fourier transform operator. We also have $l_{2}-l_{1}=\left(x_{2}-x_{1}\right) \cos \theta+\left(y_{2}-y_{1}\right) \sin \theta$, and hence the auto-correlation function $R$ is space invariant and can really be written as $R\left(d_{1}, d_{2}\right)$. This also implies that the reconstructed noise image is stationary. Furthermore, expressing $R$ in polar notation as $R(\rho, \psi)$ where $d_{1}=\rho \cos \psi$ and $d_{2}=\rho \sin \psi$, we have:

$$
\begin{aligned}
R(\rho, \psi) & =\frac{S_{0}}{4 \pi^{2}} \int_{0}^{\pi} \int_{-\infty}^{\infty}|\mathcal{H}(\omega)|^{2} e^{j 2 \pi \omega \rho \cos (\theta-\mathcal{D})} d \omega d \theta \\
& =\frac{S_{0}}{4 \pi^{2}} \int_{-\infty}^{\infty}|\mathcal{H}(\omega)|^{2} J_{0}(2 \pi \omega \rho) d \omega
\end{aligned}
$$

Hence, $R(\rho, \psi)$ is only dependent on $\rho$ (radially symmetric), the distance between two points $\left(x_{1}, y_{1}\right)$ and $\left(x_{2}, y_{2}\right)$. Intuitively, this appears reasonable. The above equation is also a zero-order Hankel transform of $|\mathcal{H}(\omega)|^{2}$ and $g_{0}(x)$ is the zero-order Bessel function of the first kind. Taking the 1-D Fourier transform of $\mathcal{R}(\rho, \downarrow)$, which really gives us the circularly symmetric noise spectrum of the $R\left(d_{1}, d_{2}\right)$ along any radial path, it is easy to show that this is proportional to $|\mathcal{H}(\omega)|^{2} / \omega$. This implies that, if a windowed version of the $1 \omega \mid$ filter is used, then the noise-power spectrum is directly proportional to this filter! This also implies that the noise has a lot of high-frequency content in it. A similar analysis [15] for fan-beam projections will show that we get the same results for the auto-correlation function as above.
- The heavy detector assembly with its lead collimators are static. The X-ray source and collimator (which is a much lighter assembly) is easier to move, improving scan times per slice.

- Circular ring artifacts were common in the older version. This was due to a poorly calibrated or malfunctioning detector in the detector array that generates a consistent data error at a particular location in $P_{\theta}(l)$ for all $\theta[20]$. The resulting reconstruction generates a spurious circular ring artifact. In the third-generation CT, such data dropouts now happen at different locations in the fan beam in only some of the projections. These dropouts will not generate the structured ring artifacts.

Considering the fan-beam geometry from Figure 11. the source subtends an angle $\beta$ with the $y$-axis. The fan beam has a maximum angle of $2 \gamma_{m}$, and each ray in the beam subtends an angle $\gamma$ with the source-origin line. We now have the relations $\theta=\beta+\gamma$ and $l=D \sin \gamma$, where $D$ is the distance from the source to the origin. The uniform sampling coordinate system is now $(\beta, \gamma)$ and the $J$ acobian $J$ evaluates to $D$ cos $\gamma$. In polar coordinates, the reconstruction of $f$ can now be written as: 


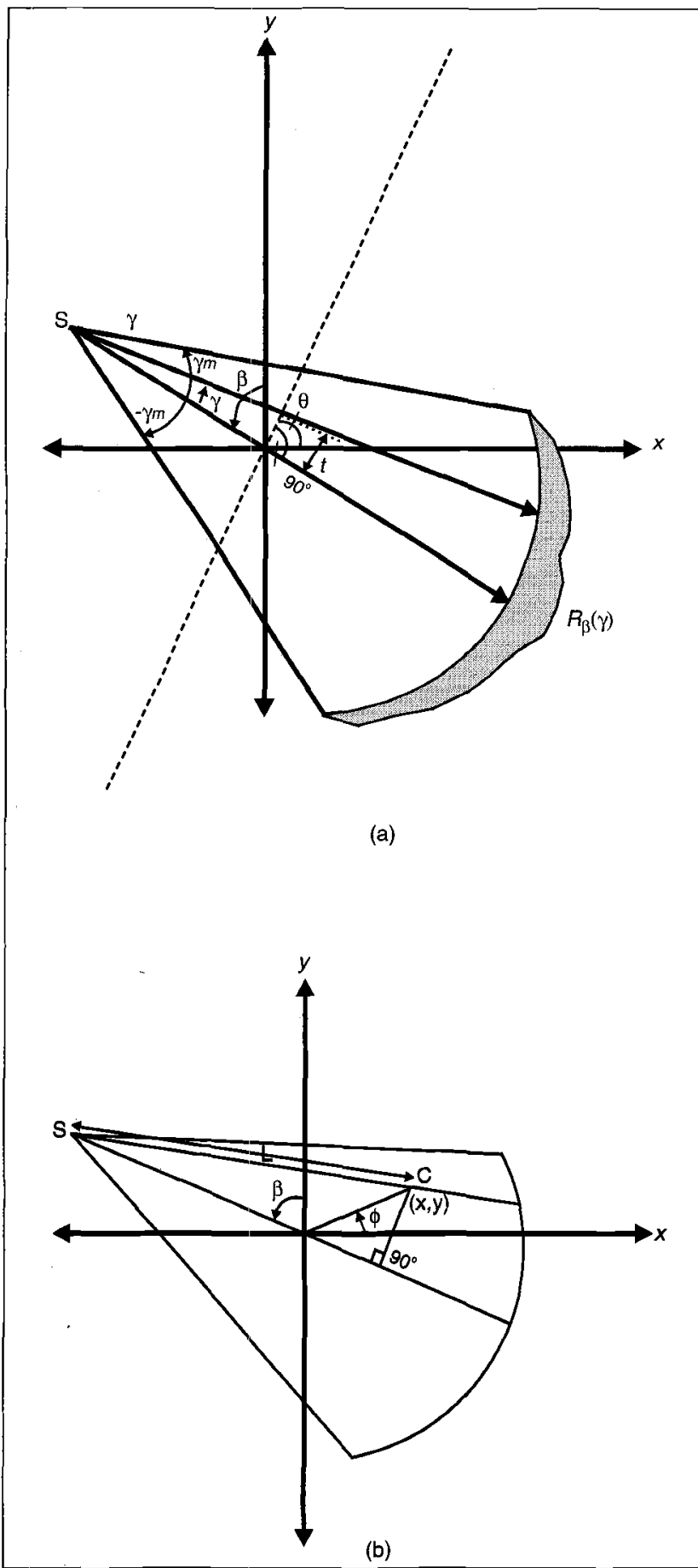

11. (a) The source, $S$, makes an angle, $\beta$, with the $y$ axis. Each individual ray subtends an angle, $\gamma$, from the central ray. The maximum angular displacement $\gamma_{m}$. (b) Back-projection after filtering. $C$ denotes the point $(x, y)$ in the path of the back-projection ray and it is at a distance, $L$, from the source, $S$.

$f(r, \phi)=\frac{1}{4 \pi^{2}} \lim _{\epsilon \rightarrow 0}$

$\int_{0}^{2 \pi} \int_{\gamma m}^{\gamma m} P_{\beta+\gamma}(D \sin \gamma) H_{\epsilon}(r \cos (\beta+\gamma-\phi)-D \sin \gamma) D \cos \gamma d \gamma d \beta$

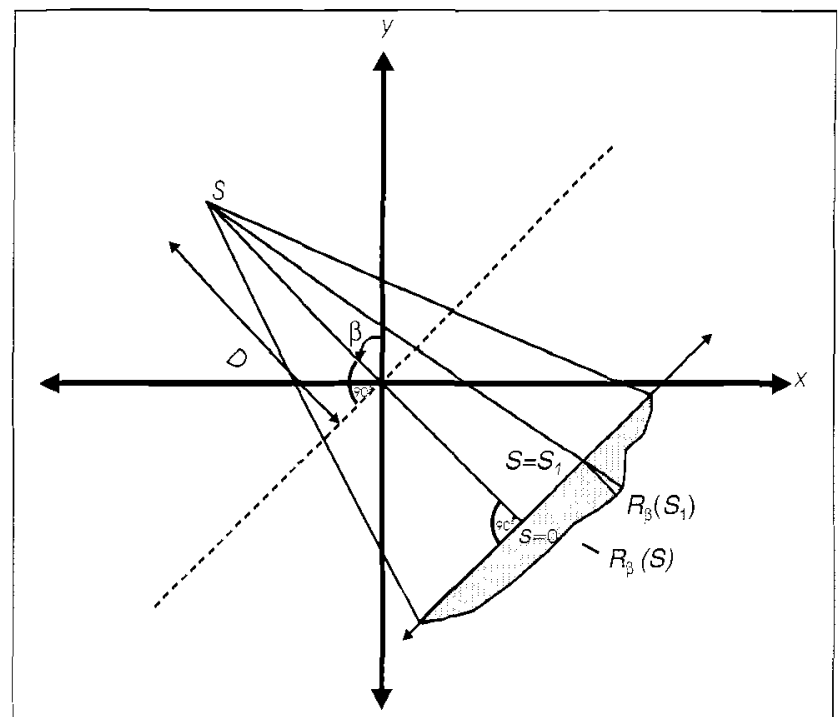

12. Equidistant detector fanbeam: The central ray makes an angle, $b$, with the y axis. The ray hitting the detector array at a distance $s=s_{1}$ from the central ray makes an angle, $\gamma$. The sampling is uniform along the collinear detector array.

where $\gamma_{m}=\sin ^{-1}\left(t_{m} / D\right)$. Let us denote $P_{\beta}+\gamma(D \sin \gamma)$ as $R_{\beta}(\gamma)$. Let $L$ be the distance of the source from $(x, y)$ (or $(r, \phi)$ in polar coordinates). The ray passing through this point subtends an angle $\gamma^{\prime}$. From Figure 11, it is easy to show that:

$$
\begin{aligned}
& L \cos \gamma^{\prime}=D+r \sin (\beta-\phi) \\
& L \sin \gamma^{\prime}=r \cos (\beta-\phi)
\end{aligned}
$$

Using these relationships, we have

$$
\begin{aligned}
& f(r, \phi)=\frac{1}{4 \pi^{2}} \lim _{\xi \rightarrow 0} \\
& \int_{0}^{2 \pi} \int_{-m}^{i m} R_{\beta}(\gamma) H_{\epsilon}\left(L \sin \left(\gamma^{\prime}-\gamma\right)\right) D \cos \gamma d \gamma d \beta
\end{aligned}
$$

The above equation looks very close to a convolution, but is really not so, since $H_{\xi}$ is not directly related to $\gamma$. By considering $H_{\epsilon}$ as a $|\omega|$ filter and using $\omega^{\prime}=\omega L \sin \gamma / \gamma$, we can easily show:

$$
H_{\epsilon}(L \sin \gamma)=\left(\frac{\gamma}{L \sin \gamma}\right)^{2} H_{\varepsilon}(\gamma)
$$

Introducing a new variable, $g(\gamma)=\left(\frac{\gamma}{\sin \gamma}\right)^{2} H_{\varepsilon}(\gamma)$, we now have the reconstruction equation as:

$$
f(r, \phi)=\frac{1}{4 \pi^{2}} \int_{0}^{2 \pi} \frac{1}{L^{2}} \int_{-\gamma m}^{\gamma m} R_{\beta}(\gamma) g\left(\gamma^{\prime}-\gamma\right) D \cos \gamma d \gamma d \beta
$$

The inner integral above is a convolution of weighted projection $R_{3}(\gamma)$ (with weighting factor $D \cos \gamma$ ) and the filter $g(\gamma)$ which is again, a weighted version of the $|\omega|$ filter. The outer integral is a weighted back-projection integral. The construction algorithm hence consists of three steps: 


\section{Spiral CT}

In conventional fan-beam CT, a series of slices are gathered by having the patient (object) translate a few millimeters between two slice acquisitions. This is a very slow process. It typically takes one second for one slice data acquisition and tens of seconds to prepare for the next data capture. In many cl nical tests (such as the thorax), the patient is required to hold his breath during slice acquisition to minimize motion artifacts. However, the degree of aspiration (breath hold) is not uniform for all slices and hence there are going to be a lot of artifacts during 3-D volume reconstruction. This standard method also precludes the use of CT in angiographic applications.

The collimators placed for the source and detector array theoretically provide for a rectangular profile in the $z$ direction, and this rectangular profile (usually termed slice thickness) is variable from $2 \mathrm{~mm}$ to $10 \mathrm{~mm}$. In practice, the profile is not rectangular due to geometrical unsharpness and X-ray scatter. The slice thickness is usually defined to be the full width at half maximum (FWHM) or full width at one-tenth naximum (FWTM) of the slice isocenter.

In spiral CT, there is continuous rotation of the source assembly around the detector ring, which is accomplished using slip-ring technology. Simultaneously, there is a constant translatory motion of the gantry in the $z$ coordinate ( $x$ and $y$ being defined in the plane of the slice) (see Figure 16). In this method, it now possible to scan an entire region (such as the thorax or abdomen) is one breath hold (less than 1 minute). The pitch of a helical scan has a bearing on the sensitivity of the slice profile and is defined as the ratio of the distance traversed by the gantry for one $360^{\circ}$ rotation to the nominal collimation aperture along the $z$ direction. Usually, the pitch will be around unity. Fractional pitches result in significant increase in X-ray doses without concomittant increases in slice profiles, while pitches with greater unity significantly degrade the slice profile $[3,13,41]$.

The projection data, $R_{\beta}(\gamma)$, is now dependent on $z$ as well. Denoting this dependence by $R_{z}(\beta, \gamma)$, let us now consider the reconstruction of a slice at $z=z_{h}$. Let us also consider a source angle, $\beta_{1}$. It is quite possible that there is no measurement for $\beta_{1}$ for $z=z_{k}$. Hence, we will have to estimate the fan-beam projection data, from neighboring measurements around $z_{h}$ that contain the fan-beam data for $\beta_{1}$. Also, let $\beta_{h}$ be the source angle for which there is measurement at $z_{b}$.

\section{Full Scan with Interpolation}

In this method [5], the data is acquired for a range of $4 \pi$ radians. A set of data for $\beta$ in the range $[0,2 \pi)$ for the $z=z_{h}$ plane is estimated. Let us estimate the projection data for $\beta$, in the $z_{h}$ plane. Obviously, we do not have a measurement for $\beta_{1}$ (unless $\beta_{1}=\beta_{h}$ ). Consider the measurements available for $\beta_{1}$ on both sides of the plane. Let $z_{\alpha}$ be the nearest gantry position on one side for which there is a measurement for $\beta_{1}$, Let $z_{b}$, be the next closest position for which there is a measurement. This position will lie on the opposite side of $z_{h}$, opposite to that of $z_{u}$. It is obvious that there are $360^{\circ}$ of fan-beam measurements between $z_{a}$ and $z_{b}$ with $z_{a} \leq z_{h} \leq z_{i}$ and $\beta_{h} \in\left[\beta_{1}, \beta\right.$ $+2 \pi]$. It is then easy to estimate:

$$
\left.R_{z h t} \beta_{1}, \gamma_{j}\right)=w_{1} R_{z u}\left(\beta_{l}, \gamma_{j}\right)+w_{2} R_{z t}\left(\beta_{l}, \gamma_{l}\right)
$$

where $w_{1}=\left(\beta_{1}+2 \pi-\beta_{h}\right) / 2 \pi$ and $w_{2}=\left(\beta_{h}-\beta_{1}\right) / 2 \pi$. This is essentially linear interpolation. Hence, all the projection data for the range $\beta \in[0,2 \pi)$ for $z_{h}$ is estimated before applying the standard fan-beam back-projection algorithm. Note that a measurement set over $4 \pi$ range is needed to estimate all the data over a $2 \pi$ range in the $z=z_{h}$ plane.

Since this backprojection is a linear operation, an alternative is to pre-multiply the data with an interpolation weight. $w(\beta, \gamma)$, and perform filtered back-projection on the entire set of data covering a $4 \pi$ range. This weight is given by:

$$
w(\beta, \gamma)=\left\{\begin{array}{cc}
\frac{\beta}{2 \pi} & 0 \leq \beta \leq 2 \pi \\
\frac{4 \pi-\beta}{2 \pi} & 2 \pi \leq \beta \leq 4 \pi
\end{array}\right.
$$

\section{Half-Scan Methods}

In the standard fan-beam algorithm, we argued that we really need data only for $\beta \in\left[0, \pi+2 \gamma_{m}\right]$. The overlapped measurements are compensated by using a smoothed (continuous and differentiable) one-zero weighting filter in the reconstruction algorithm. Using this concept, it is easy to see that reconstruction can be achieved with less than $4 \pi$ views. In fact, we need on $2 \pi+4 \gamma_{m}$ views. In this case, the weighting factor $w(\beta, \gamma)$ are slightly complex and their formulation is beyond the scope of this discussion. Refer to [5] for more details. This method is called the half-scan with interpolation method. There are two benefits to this method. First, a lesser number of views are required. Second, there is less table motion per slice, and hence the slice profile will be much better.

In the half-scan with extrapolation method [5], the data is acquired for only $2 \pi$ views. In this case, for nonredundant data, interpolation is used. For data in the redundant region, extrapolation of data on the same side of the plane $z_{h}$ is used. It has been found that this extrapolation causes no degradation in the reconstruction. The advantage of this method is that only $2 \pi$ views are required. The weighting factor, $w(\beta, \gamma)$, is discussed in detail in [5].

Spiral CT has implications in volumetric analysis. In standard CT, the sampling in the $z$ direction is not the same as in the $(x, y)$ plane, and this leads to gross errors in volume reconstructions. Using spiral $C T$, true isotropic sampling [21] can now be attempted, and this leads to better multiplanar reformating (MPR) [38] and volume visualization. For instance, in Cranio/Maxilio-facial reconstructive surgeries $[38,18]$, such isotropic sampling reduces artifacts and volumetric errors. 
1. The projection data, $R_{\beta}(\gamma)$, is weighted with $D \cos \gamma$ to obtain $R_{\beta}^{\prime}(\gamma)$. The sampled version is denoted as, where $\alpha$ is the angular sampling interval.

2. The weighted data, is then convolved with the weighted filter:

$$
g(n \alpha)=\left(\frac{n \alpha}{\sin n \alpha}\right)^{2} H_{\mathrm{e}}(n \alpha)
$$

$H_{\epsilon}(n \alpha)$ is any of the back-projection filters we discussed previously and this may be windowed (with a windowing filter such as a Hamming window). This filtering gives us the intermediate result $Q_{\beta}(\gamma)$.

3. The data in the sampled $(x, y)$ domain can then be reconstructed using:

$$
f(i \Delta x, j \Delta y)=\frac{\Delta \beta}{4 \pi^{2}} \sum_{k=1}^{M} \frac{1}{L^{2}\left(i \Delta x, J \Delta y, \beta_{k}\right)} Q_{\beta_{i}}\left(\gamma^{\prime}\right)
$$

where $\gamma$ is angle that the ray passing through $(i \Delta x, j \Delta y)$ makes with the source-origin line. Obviously, this ray may lie between two other rays in the projection data and suitable interpolation (as discussed before) will have to be employed to obtain the interpolated $Q_{\beta k}(\gamma)$.

\section{Fan-Beam Projections with Equidistant Collinear Detectors}

In this configuration, the detectors are placed in a linear array (see Figure 12). This setup is very popular in industrial $\mathrm{CT}$ for two reasons. First, in industrial CT, it is easier and more convenient to have the object rotating about the axis, rather than have the source detector assembly moving around the object. Second, it is easier to place the detectors in a linear array than along a ring.

Even though the detector array is usually at a distance from the origin, we may consider the detector array to be placed on a straight line passing through the origin (Figure 12). Let $s$ be the distance along this line from the origin. In this geometry, uniform sampling is done in the $(\beta, s)$ domain and its relationship with the $(x, y)$ domain is given by:

$$
l=\frac{D s}{\sqrt{D^{2}+s^{2}}} \text { and } \theta=\beta+\tan ^{-1} \frac{s}{D}
$$

$l$ is independent of $\beta$, but has a highly nonlinear relationship with $s$. The Jacobian $J$ is found to be and the reconstruction equation can be written as:

$$
\begin{aligned}
& f(r, \phi)=\frac{1}{4 \pi^{2}} \int_{0}^{2 \pi} \int_{-s_{m}}^{s_{m}} R_{\beta}(s) H_{\varepsilon} \\
& \left(r \cos \left(\beta+\tan ^{-1} \frac{s}{D}-\phi\right)-\frac{D s}{\sqrt{D^{2}+s^{2}}}\right) \frac{D^{3}}{\left(D^{2}+s^{2}\right)^{3 / 2}} d s d \beta
\end{aligned}
$$

Using the relationships $\beta_{1}-\gamma_{1}=\beta_{2}-\gamma_{2}-180^{\circ}$ and $\gamma_{1}=-\gamma_{2}$ and noting that the fan-beam angle $\gamma$ is always less than $90^{\circ}$, it is easy to show that the overlapped regions are given by:

$$
\begin{aligned}
& 0 \leq \beta_{2} \leq 2 \gamma_{m}+2 \gamma_{m} \\
& 180^{\circ}+2 \gamma_{2} \leq \beta_{1} \leq 180^{\circ}+2 \gamma_{m}
\end{aligned}
$$


In [20], an example is demonstrated for the reconstruction from $180^{\circ}+2 \gamma$ projections without any correction for the overlapped data. Naperstek [25] shows that the usage of a one-zero window filter, which essentially zeroes out data in one of the regions of the overlap, gives only a marginal improvement. However, in [27] the author demonstrates the results of using a smoother window that is continuous and has a continuous derivative. The results are indistinguishable from a full $360^{\circ}$ reconstruction. The poor results for the one-zero window is due to the fact that the sharp cutoff of the one-zero window introduces high frequencies that are amplified by the $|\omega|$ filter.

The advantages of reconstructions from limited views are obvious. There will be less exposure to ionizing radiation and faster data acquisition leading to fewer patient-motion artifacts. Figure 14 shows some typical slice reconstructions.

\section{Cone-beam CT}

In 3-D, the Radon transform is obtained by integrating along planes. If we represent a plane by $(\beta, l)$, where $\beta$ is a unit 3-D vector in a unit sphere representing the orientation of the plane with respect to the coordinate axes and $l$ represents the distance of the plane from the origin, then we consider all planes that intersect the object and obtain the planar integral of $f(x, y, z)$ along that plane. The obtained result gives us the Radon transform data in 3-D. However, in practice, we only have the line integral $g$ through an object. How do we obtain the line-integral data? In fan-beam tomography, only a thin slice (or plane) of X-rays, diverging from a point source, is permitted to penetrate the object by placing a suitable collimator in front of the source. If this collimator is removed, a divergent cone beam will emerge from the X-ray source and penetrate the object. The line-integral data through the object is collected on the opposite side of the source by a 2-D array of detectors. The source-detector array assembly will traverse along a suitable locus, and measurements are captured at various points along the trajectory to get a set of projection data. If the source locus is a complete trajectory, then the projection data set is complete and the object can be completely recovered. The completeness of a trajectory is discussed in later in this article.

\section{Some Definitions for 3-D Reconstructions}

Let $f(\mathbf{x})=f(x, y, z)$ denote the object. The support for the object $f(\mathbf{x})$ is a ball of radius $R$ in $R^{3}$. Let $\beta$ be a unit vector in $R^{3}$, i.e., $\beta_{\theta}, \phi=(\cos \phi \sin \theta, \sin \phi \sin \theta, \cos \theta)^{T}$, where $\theta$ and $\phi$ are elevation and azimuth angles in spherical coordinates. The vector $\beta_{\theta, \phi}$ may also be represented as $\beta_{\theta, \phi}=\left(\beta, \beta_{\perp 1}, \beta_{\perp 2}\right)$ where $\beta$, $\beta_{\perp 1}$, and $\beta_{\perp 2}$, are orthonormal. We shall drop the $\theta, \phi$ subscript on $\beta$ without any loss of generality. In $3-D$, the Radon transform is defined as:

$$
\breve{f}(\beta, l)=\int_{\mathbb{R}^{3}} f(\mathrm{x}) \delta(l-\beta \cdot \mathrm{x}) d \mathrm{x}
$$

where $l$ is the perpendicular distance from the origin to the plane of integration, and $\beta$ is a unit vector along $l$ that defines the orientation of the plane. $\breve{f}(\beta, l)$ must be known over all $(\beta, l)$ for the data to be complete. The $3-\mathrm{D}$ inverse Radon transform can be shown to be $[4,7,34]$ :

$$
f(x)=-\frac{1}{(2 \pi)^{2}} \int_{0}^{\pi} \int_{0}^{\pi} \frac{\delta^{2}}{\delta l^{2}} \tilde{f}(\beta, x \cdot \beta) \sin \theta d \theta d \phi
$$

The measured data, $g$, is not the Radon transform data, $\tilde{f}(\beta, l)$, since the X-rays that penetrate the object are line integrals and not planar integrals. We need to convert the measured line-integral data to Radon transform data. In order to do so, we define an intermediate representation. This function can be derived from the Radon transform as well as the lineintegral data and is then used to reconstruct the original object $f(\mathbf{x})$. First we define $[32,33]: \mathbb{P}(\beta, l)=\lim _{\varepsilon \rightarrow 0} H_{\epsilon}(l-t) d t$ where $H_{\epsilon}(t)$ is the kernel defined earlier. Since the support of $f(\mathbf{x})$ is a sphere of radius $R$, we define $F$ as the restriction of $P$ on the set, i.e., $F$ is defined over the domain $S^{2} / 2 \times[-R, R]$, where $S^{2} / 2$ is a unit hemisphere. Defrise et al. [9] examined the errors that arise out of such restrictions on $P$. We know that $\lim _{\epsilon \rightarrow 0} \int_{R} H_{\epsilon}(l-\tau) h(\tau) d \tau=\mathscr{F}_{\omega}^{-1}\{0 \mid \widetilde{h}(\omega)\}$ and, using the central slice theorem, it is easy to show $\frac{1}{\pi} \frac{1}{l^{2}} * F(\beta, l)=\frac{\delta^{2}}{\delta l^{2}} \breve{f}(\beta, l)$. If $F$ is known on its entire domain, we can reconstruct $f$ using:

$$
f(x)=\frac{-1}{4 \pi^{2}} \int_{0}^{\pi} \int_{0}^{\pi} \int_{-\infty}^{\infty} \frac{1}{(l-t)^{2}} F(\beta, l) d t \sin \theta d \theta d \phi
$$

Relationship Between the Cone-Beam Data and F $(\beta, l)$ The source trajectory always lies outside the support of the object $f(\mathbf{x})$. Denoting the source position by the vector $\Phi$, then the line integral (the vector $\alpha$ gives the direction of each ray) is nonzero only along the rays, leaving $\Phi$ within a cone that covers the support of the object. The line integral is then given by $[32,36,37]$ :

$g(\alpha, \Phi)=\int_{-\infty}^{\infty} f(\Phi+t \alpha) d t=\frac{1}{|\alpha|} \int_{-\infty}^{\infty} f\left(\Phi+t \frac{\alpha}{\alpha \mid}\right) d t ; \forall \alpha \in \mathbb{R}^{3}$

By defining $\alpha$ over $R^{3}$ rather than $S^{2} / 2$ we are able to consider the Fourier transform of $g(\alpha, \Phi)$ for fixed $\Phi$ :

$$
\begin{aligned}
& G(\beta, \Phi)=\int_{R^{3}} g(\alpha, \Phi) e^{-\alpha \cdot 3} d \alpha \\
& =\int_{-\infty}^{\infty} \int_{\pi^{3}} f(\Phi+t \alpha) e^{-j \alpha \cdot \beta} d \alpha d t
\end{aligned}
$$

Performing a change of variables $v=\Phi+t \alpha$. followed by substitution of $t$ by $1 / \tau$ it is easy to show that: 


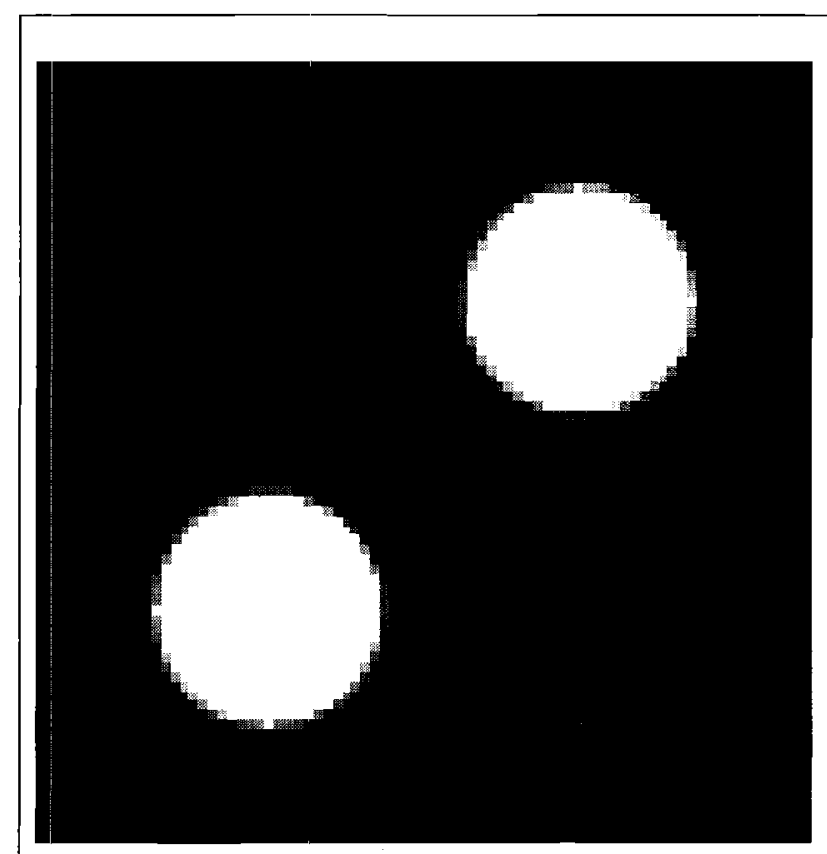

(a)

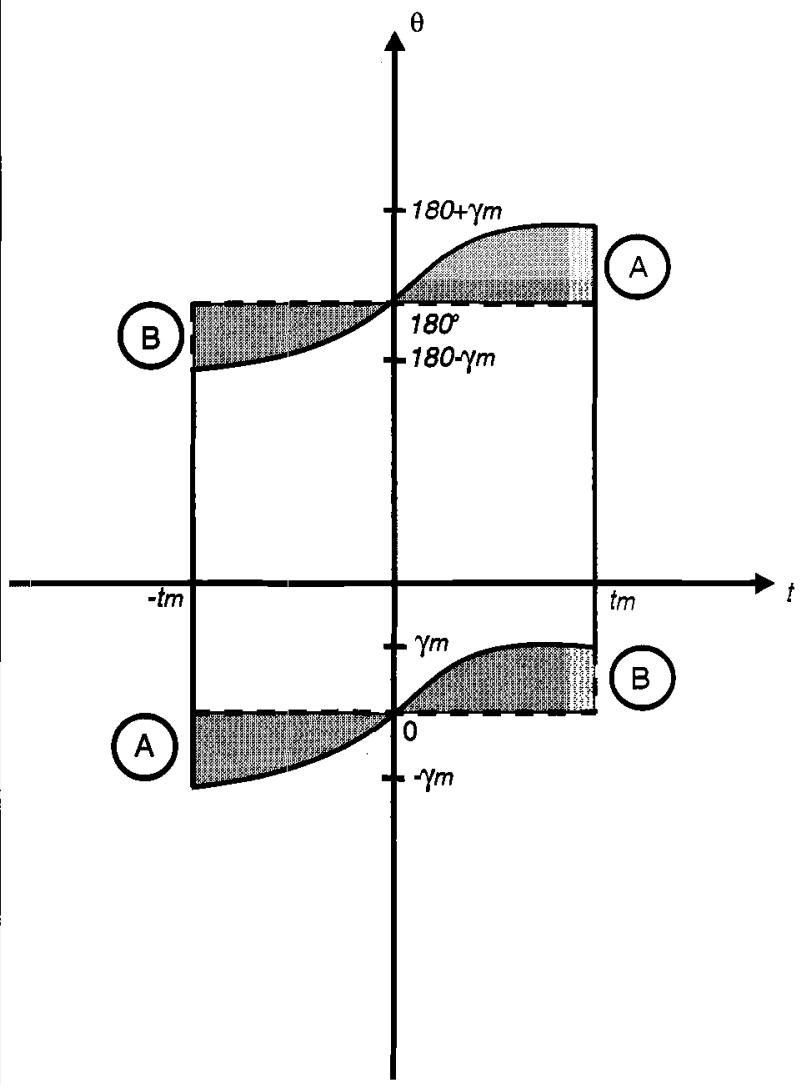

(c)

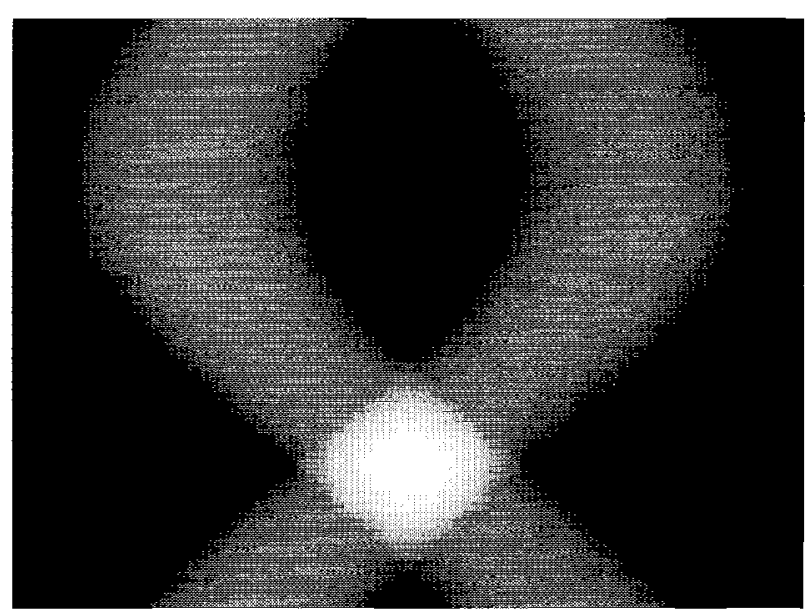

(b)

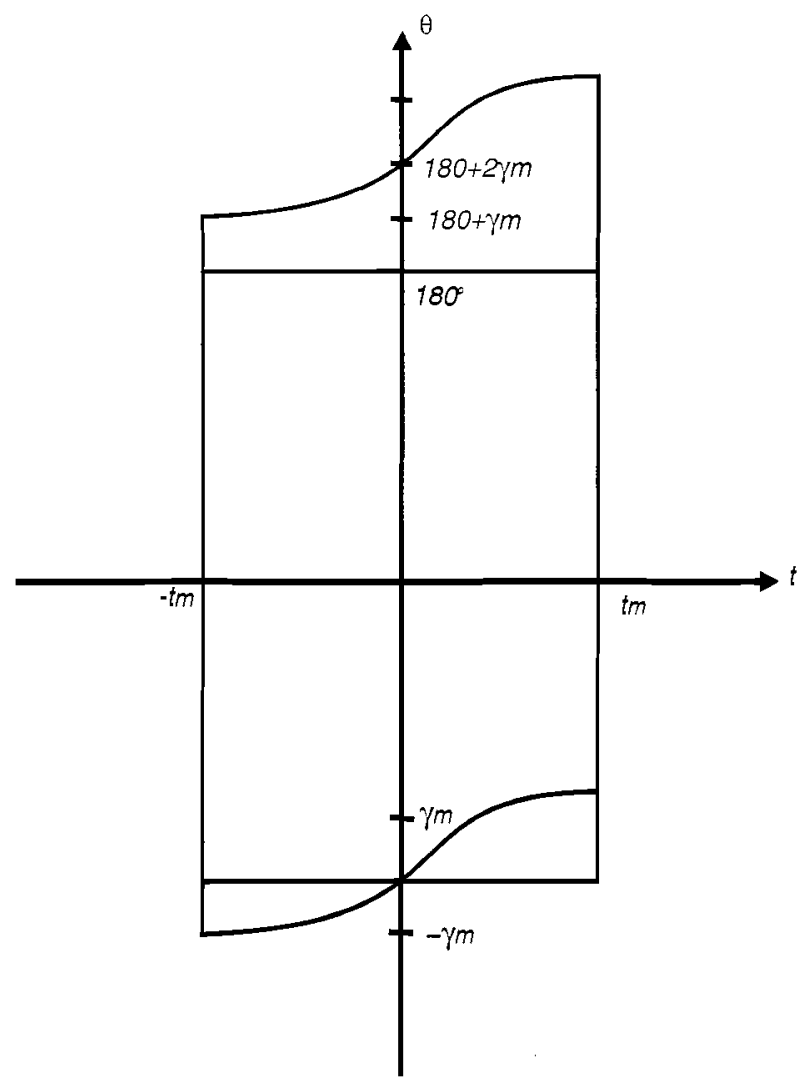

(d)

13. (a) An image, $f(x, y)$, and (b) its sinogram (reprinted with permission from J.L. Prince et al. [24]). (c) This figure demonstrates limited-view fan-beam reconstruction. If $\beta$ ranges only from 0 to $180^{\circ}$, then we have duplication of data in region $A$, while there is no data available in region $B$. (d) If we increase the scanning angle to $180+2 \gamma_{m}$, then we will be covering the entire $(l, \theta)$ range. However, the duplication of data will occur over a larger region. 


$$
G(\beta, \Phi)=2 \pi F(\beta, \Phi \cdot \beta)
$$

The object $f(\mathbf{x})$ can now be constructed from the $g(\alpha, \Phi)$. The relationship graph between $f, \breve{f}, F, g$, and $G$ is shown in Figure 15. Since $g(\alpha, \Phi)$ is a slowly increasing function, we have to consider $G(\beta, \Phi)$ as a generalized Fourier transform of $g(\alpha, \Phi)[37]$.

\section{Computing $G$ and $F$}

The various stages in the reconstruction and associated issues are discussed below:

- Computation of $G(\beta, \Phi)$ from $g(\alpha, \Phi))$ : The Fourier transform of $g(\alpha, \Phi)$ may not exist and its computation using the DFT may result in errors [32]. It has been found that much of the degradation during reconstruction occurs at this stage [35].

- Computation of $F(\beta, l)$ from $G(\beta, \Phi)$ : To compute $f(\mathbf{x})$ from $F(\beta, l)$ it is desirable to have values of $F(\beta)$ at uniform increments of $l$ for each $\beta$, which, in turn, uniformly sam- pled in $\theta$ and $\phi$. Since $G(\beta, \Phi)$ is known only at a finite number of $\Phi$, an interpolation is needed to obtain $F(\beta, l)$ on a uniform grid. One method is to use the linear interpolation [35]:

$$
F\left(\beta, l_{i}\right)=\frac{1}{2 \pi}\left\{G\left(\beta, \Phi_{j}\right) \frac{l_{i}-l_{k}}{l_{j}-l_{k}}+G\left(\beta, \Phi_{k}\right) \frac{l_{i}-l_{j}}{l_{k}-l_{j}}\right\}
$$

where $\Phi_{j}$ and $\Phi_{k}$ are known, and $l_{j}=\beta \cdot \Phi_{j}$ and $l_{k}=\beta \cdot \Phi_{k}$. While the samples, $\Phi_{k}$, may be uniformly sampled in their domain, the corresponding $l_{k}=\beta \cdot \Phi_{k}$ is not uniformly spaced. Also, linear interpolation may not be the best way to calculate $F(\beta, l)$. Lagrange interpolation [17] can be expected to perform better than linear interpolation because uniformly spaced pivotal points are not needed. Cubic splines may also be considered since they are computationally more efficient than Lagrange methods.
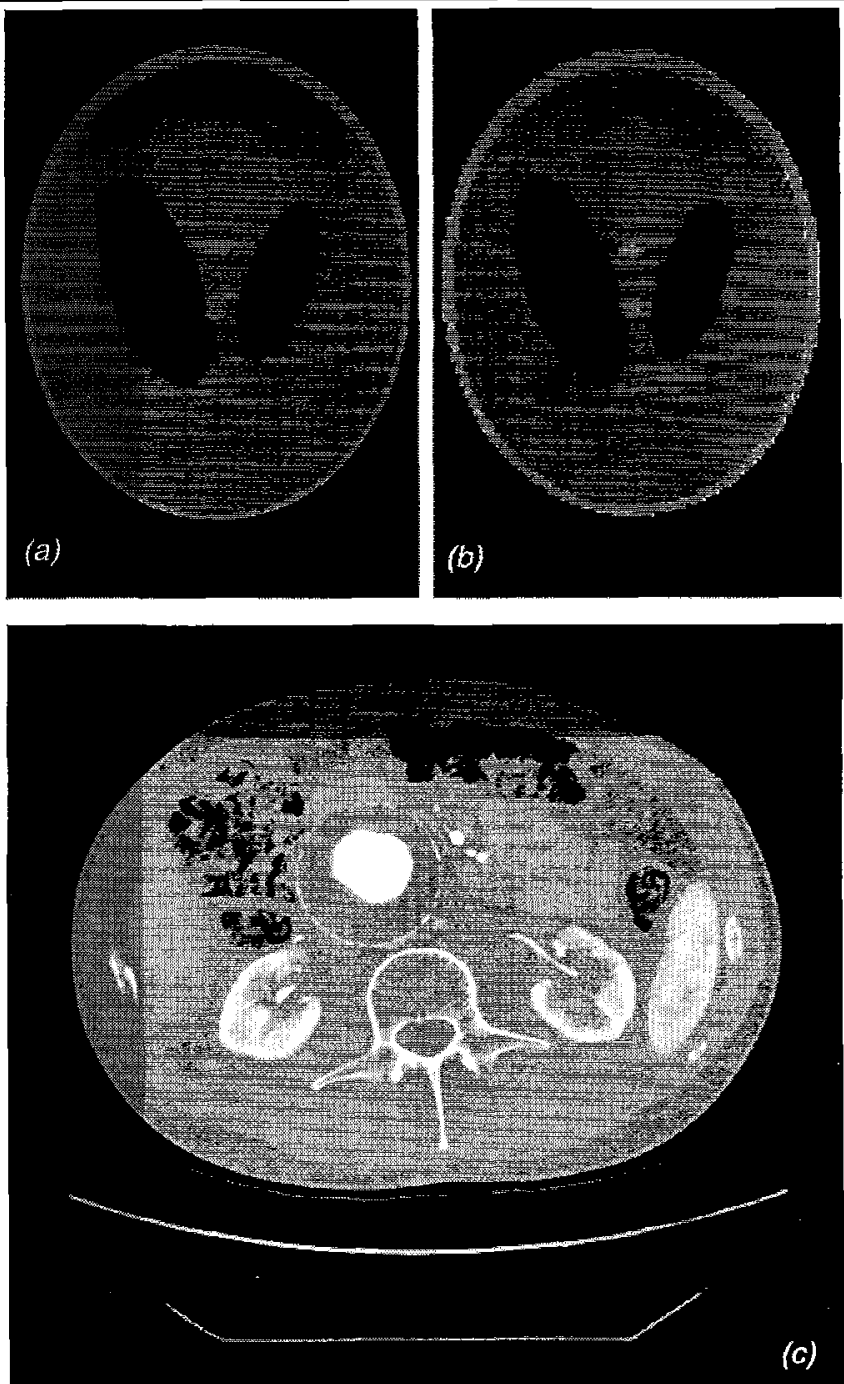

14. Reconstructed images. (a) Shepp-Logan head phantom. (b) Reconstruction of the head phantom from parallel beam projections. (c) Typical CT fan-beam reconstruction of an abdominal section ( $(a)$ and (b) have been reprinted with permission from Kak and Rosenfeld [19]). 


\section{Completeness of Data}

$f(\mathbf{x})$ can be reconstructed if $F(\beta, l)$ can be determined on its whole domain. $F(\beta, l)$ can be determined from $G(\beta, \Phi)$ (and hence from $g(\alpha, \Phi)$ ) if, for each direction, $\beta$, there exists a source point, $\Phi$, such that $\beta \cdot \Phi=l$. The locus of the source point that satisfies $\beta \cdot \Phi=l$ gives a complete geometry and artifact-free reconstruction is possible. Since $\|\beta\|=1$, the length $l$ predominantly comes out of the norm of $\Phi$ denoted by $\|\Phi\|$, and the angle between $\beta$ and $\Phi$. This condition implies that: "If on every plane that intersects the object there lies a vertex (source point), then one has complete information about the object." The above condition is the same as Tuy's [37]. Some examples of such complete trajectories are: three twists of a helix, two periods of sine on a cylinder, twin orthogonal circles, baseball seam curve, etc. The standard circular trajectory used in 2-D tomography is obviously incomplete, and various analytical continuation methods $[23$, 14] have been used to obtain reasonable reconstructions from incomplete data.

\section{Other Reconstruction Methods in Cone-beam CT}

In the discussion on cone-beam CT reconstruction, we have concentrated exclusively on B.D. Smith's method. In reality, there are three distinct methods due independently to Tuy [37], Smith [33] and Grangeat [12]. All of these methods have been derived from the original work by Kirillov [22], which deals with cone-beam reconstruction from an $n$-dimensional complex space. Defrise and Clack [8] derive a very elegant result that integrates all three methods. We will now consider briefly Grangeat's results, and explain Defrise and Clack's formulas. Let us consider the 3-D inverse Radon transform equation:

$$
f(\mathbf{x})=-\frac{1}{(2 \pi)^{2}} \int_{0}^{\pi} \int_{0}^{\pi} \frac{\delta^{2}}{\delta l^{2}} \check{f}(\beta, \mathbf{x} \cdot \beta) \sin \theta d \theta d \phi
$$

It is easy to see that $\frac{\delta^{2}}{\delta l^{2}} \breve{f}(\beta, l)=\mathcal{F}^{-1}\left\{\omega^{2} \mathcal{F}[\breve{f}(\beta, l)]\right\}$. It is also to be noted that $\omega^{2}=|\omega|^{2}$ and $|\omega|_{2}$ can be easily used instead in the above equation. Smith uses the $|\omega|^{2}$ formulation in obtaining the intermediate $F$ function, while Grangeat prefers to calculate and re-bin the cone-beam data directly to $\frac{\delta}{\delta l} \breve{f}(\beta, l)$ and then calculates $\frac{\delta^{2}}{\delta l^{2}} \breve{f}(\beta, l)$ using the differential operator once more. This is equivalent to using the $\omega^{2}$ filter. Grangeat develops an elaborate method of relating the line integral in the detector plane containing the cone-beam data to the first derivative of the Radon transform. Defrise and Clack integrate these two methods along with Tuy's as follows. Let $g(\alpha, \Phi)$ be the measured cone-beam data as defined previously. Let $G(\beta, \Phi)$ be an intermediate function defined as:

$$
G(\beta, \Phi)=\int_{S^{2}} g(\alpha, \Phi)\left[a h_{1}+b h_{2}\right](\alpha \cdot \beta) d \alpha
$$

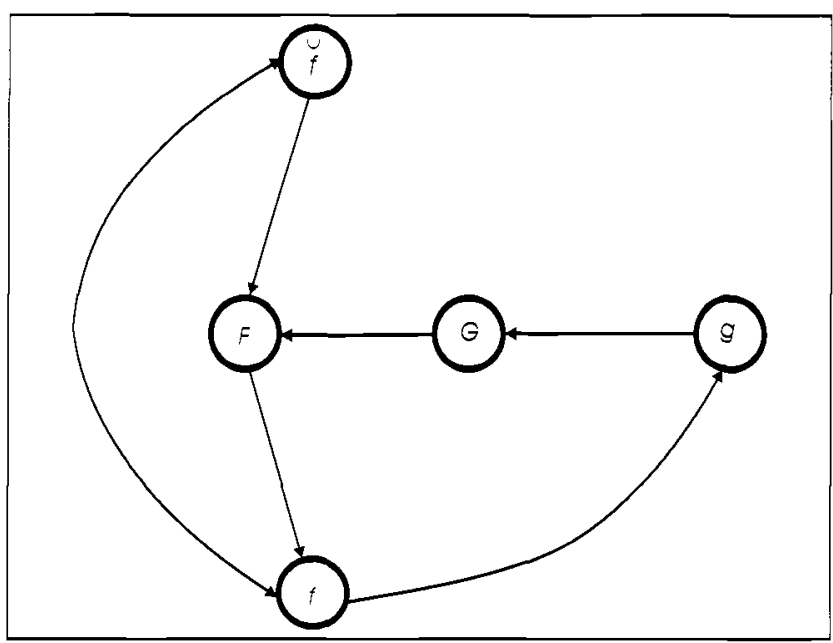

15. $f$ is the Radon transform of the 3-D object $f(x)$. F is the intermediate function. $g$ is the measured cone-beam line-integral data. To reconstruct $f$, we first calculate $G$ and then obtain $F$. $f$ is then recovered by filtering $F$ and back-projecting the result.

where $h_{1}(t)=\mathcal{F}^{1}\{|\boldsymbol{\omega}|\}$ and $h_{2}(\tau)=i \mathcal{F}^{1}\{\boldsymbol{\omega}\}$. We can derive $f(\mathbf{x})$ as follows:

$$
f(\mathbf{x})=\frac{1}{2} \int_{S^{2}} F^{*}(\beta, \mathbf{x} \cdot \beta) d \beta
$$

where $F^{*}$ is the convolution $\left.\right|_{\mathscr{R}} F(\beta, l)\left[c h_{1}+d h_{2}\right](l-t) d t$ and $F$ is given by $F(\beta, 1)=G(\beta, \Phi \cdot \beta)$. It is assumed here that $\Phi$ satisfies Tuy's (and Kirillov's) trajectory condition. Also, the relation $a c+b d=1$ must be satisfied. We obtain Smith's solution by setting $\{a, b, c, d\}=\{1,0,1,0\}$, Grangeat's solution by setting $\{a, b, c, d\}=\{0,-2 \pi, 0,-1 / 2 \pi\}$, and Tuy's solution by setting $\{a, b, c, d\}=\{1 / 2, i / 2,0,-2 i\}$.

The cone-beam reconstruction algorithm is $O\left(N^{+}\right)$and computationally very expensive. Axelsson and Danielsson [1] have developed a fast implementation of Grangeat's method in $O\left(N^{3} \log N\right)$ time using linograms [10] and direct Fourier methods. Another important approximate method for cone-beam reconstruction is due to Feldkamp [11] where the source trajectory is a circle. Wang et al. [40] have derived a generalized Feldkamp method with spiral scanning, and this method has practical scanner implementations in microtomography for spherical and rod shaped objects.

\section{Summary}

In this article, we have briefly looked at reconstruction in 2-D and 3-D tomography. We have not dealt with some of the issues in reconstruction such as sampling and aliasing artifacts, finite detector aperture artifacts, beam hardening artifacts, etc., in greater detail since these are beyond the scope of an introductory tutorial. CT as an engineering discipline is over 25 years old and the body of literature is vast. We have also not examined the 3 -D visualization issues in $\mathrm{CT}$. This topic should be dealt with separately. 
(a)

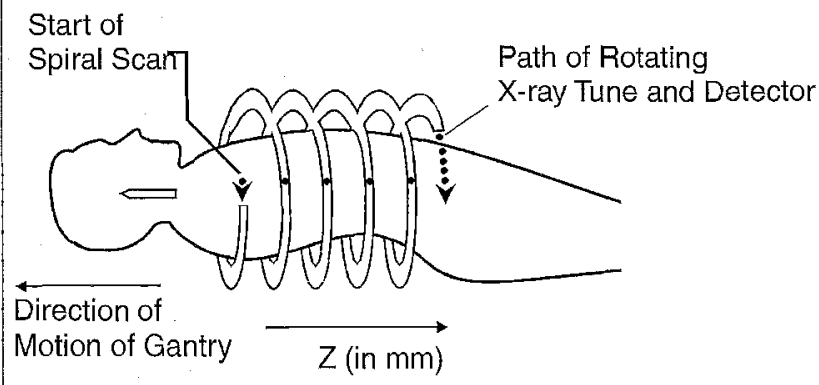

(b)

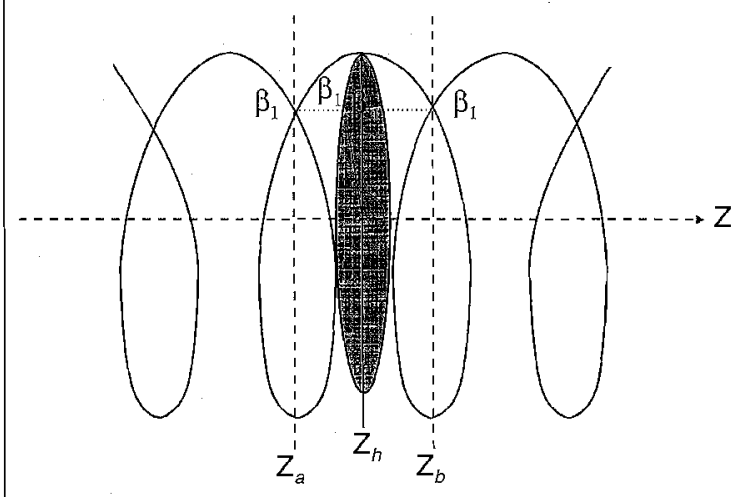

16. Spiral CT. (a) This figure shows the table motion and scanning geometry. (b) This figure demonstrates the full-scan with interpolation algorithm. At $z h$, the measured data is at angle $\beta_{h}$. In order to obtain data for $\beta_{1}$, we choose the next available helical position on both sides of $z h$, which has data for $\beta_{1}$. These positions are $z_{a}$ and $z_{b}$. The data for $\beta_{l}$ at $z_{h}$ is then interpolated between the measured data at $z_{a}$ and $z b$

Harish P. Hiriyannaiah is a project manager at InfoGain, Inc., in Cupertino, California. He can be reached at harish@infogain.com.

\section{References}

1. C. Axelsson and P. Danielsson. Three-dimensional Reconstruciton from Cone-beam Data in $o\left(n^{3} \log n\right)$ Time. Physics in Medicine and Biology, pages 477-491, 1994.

2. R.N. Bracewell. Strip Integration in Radio Astronomy. Aust. J. Physics, 9:198-217, 1956.

3. J.A. Brink, J. P. Heiken, G. Wang, McEnery K, W., Schlueter F. J., and M. W. Vannier. Helical CT: Principles and Technical Considerations. Radio Graphics, 14(4):887-893, July 1994.

4. R. Courant and D. Hubert. Methods of Mathematical Physics, volume 1. Interscience, New York, 1953.

5. C.R. Crawford and K.F. King. Computed Tomography Scanning with Simultaneous Patient Translation. Medical Physics, 17(6):967-982, Nov/Dec 1990.

6. R.E. Crochiere and L.R. Rabiner. Mulitrate Digital Signal Processing. Prentice Hall, NJ, 1983.

7. S.R. Deans. The Radon Transform and Some of Its Applications. John Wiley, 1983.
8. M. Defrise and R. Clack. Cone-beam Reconstruction by the Use of Radon Transform Intermediate Functions. Journal of the Optical Society of America A, 11(2):580-585, February 1994.

9. M. Defrise, R. Clack, and R. Leahy. A Note on Smith's Reconstruction Algorithm for Cone Beam Tomography. IEEE Transactions on Medical Imaging, 12(3):627-628, September 1993

10. P.R. Edholm and G.T. Herman. Linograms in Image Reconstruction from Projections. IEEE Transactions on Medical Imaging, 6(4):301-307. December 1987.

11. L.A. Feldkamp, L.C. Davis, and J.W. Kress. Practical Cone-beam Algorithm. Joumal of the Optical Society of America A, 1(6):612-619, June 1984.

12. P. Grangeat. Mathematical Framework of Cone Beam 2-D Reconstruction via the First Derivative of the Radon Transform. Lecture Notes in Mathematics, 1497(2):66-97, 1991.

13. J.P. Heiken, J.A. Brink, and M.W. Vannier. Spiral (Helical) CT. Radiol ogy, 189(3):647-656, December 1993.

14. H.P. Hiriyannaiah, M. Satyaranjan, and K.R. Ramakrishnan. Reconstruction from Incomplete Data in Cone beam Tomography. Optical Engineering, 35(9):2748-2760, Sept 1996.

15. H.P. Hiriyannaiah, W.E. Snyder, and G.L. Bilbro. Noise in Reconstructed images in Tomography: Parallel, Fan and Cone beam Projection. In Proc. IEEE Conference on CBMS, Chapel Hill, NC, June 1990.

16. B.K.P. Horn. Density Reconstruction Using Arbitrary Ray-Sampling Schemes. Proceedings of the IEEE, 66(5):551-562, May 1978

17. R. Horubeck. Numerical Methods. Jong Guang Co., Taiwan, 1981

18. H. Fuchs, K.H. Hoehne, and S.M. Pizer, editors. $3 D$ Imaging in Medicine: Algorithms, Systems, Applications. Springer Verlag, 1990.

19. A.C. Kak and A. Rosenfeld. Digital Picture Processing, volume 1. Academic Press, 2nd edition, 1982

20. A.C. Kak and M. Slaney. Principles of Computerized Tomographic Imaging. IEEE Press, 1988.

21. W.A. Kalendar. Thin-Section Three-dimensional Spiral CT: Is Isotropic Imaging Possible? Radiology, 197(3):578-580, December 1995.

22. A.A. Kirillov. On a Problem of I.M. Gelfand. Sov. Math. Doki., 2:268$269,1961$.

23. H. Kudo and T. Saito. Tomographic Image Reconstruction from Incomplete Cone Beam Projections by the Method of Convex Projections. Electronics and Communication in Japan, Part 3, 74(9):54-62, January 1991

24. J.L. Prince and A.S. Willsky Hierarchical Reconstruction Cusing Geometry and Sino-gram Restoration. IEEE Transactions on Image Processing, 2(3):401-416, July 1993.

25. A. Naperstek. Short-scan Fan-beam Algorithms for CT. IEEE Trans on Nucl. Sci., NS-27, 1980.

26. A.V. Oppenheim and R.W. Schaffer. Digital Signal Processing. Prentice-Hall, 1975.

27. D.L. Parker. Optimal Short-scan Convolution Reconstruction for Fanbeam CT. Med. Phys., 9:254-257,1982.

28. J. Radon. Uber die Bestimmung von Funktionen durch ihre Integralwerte langs gewisser Mannigfaltigkeiten. Berichte Saechsische Akademie der Wissershcafien, 69:262-279, 1917.

29. G.N. Ramachandran and A.V. Lakshminarayanan. Three-dimensiona! Reconstruc-tion from Radiographs and Electron Micrographs: Application of Convolution Instead of Fourier Transforms. Proceedings of the National Academy of Sciences U.S., 68:2236-2240,1970.

30. S.J. Riederer, N.J. Pelc, and D.A. Chesler. The Noioise Power Spectrum in Computed X-ray .Tomography. Physics in Medicine and Biology, 23(3):446-454, 1978.

31.B.D. Smith. Derivation of the Extended Fan-beam Formula. IEEE Transactions on Medical Imaging, MI-4: 177-184,1985.

32. B.D. Smith. Image Reconstruction from Cone-Beam Projections: Necessary and Sufficient Conditions and Reconstruction Methods. IEEE I ransactions on Medical Imaging, MI-4(1):14-25, March 1985. 
33. B.D. Smith. Computer-aided Tomographic Imaging from Cone-beam Data. PhD thesis, University of Rhode Island, 1987.

34. B.D. Smith. Cone-Beam Tomography: Recent Advances and a Tutorial Review. Optical Engineering, 5:524-534, May 1990.

35. B.D. Smith and J. Chen. Implementation, Investigation, and Improvement of a Novel Cone-Beam Reconstruction Method. IEEE Transactions on Medical Imaging, MI-11(2):260-266, June 1992.

36. K.T. Smith, D.C. Solman, S.L. Wagner, and Hamaker. Mathematical Aspects of Divergent Beam Radiography. Proc. Natl. Acad.. USA, 75(5):2055-2058, May 1978.

37. H.K. Tuy. An Inversion Formula for Cone-Beam Reconstruction. SIAM Journal of Applied Mathematics, 43(3):546-551, June 1983.
38. J.K. Udupa and G.T. Herman, editors. 3D Imaging in Medicine. CRC Press, 1989.

39. S.A. Teukolsky W.H. Press, B.P. Flannery and W.T. Vetterling, Numerical Recipes in C: The Art of Scientific Computing. Cambridge Univ. Press. 1988 .

40. G. Wang, T. Lin, P. Cheng, and D.M. Shinozaki. A General Cone-Beam Reconstruction Algorithm. IEEE Transactions on Medical Imaging, 12(3):486-496, September 1993

41. G. Wang and M.W. Vannier. Helical CT Inage Noise - Analytical Results. Medical Phisics, 20(6):1635-1640, Nov/Dec 1993. 